This material is posted here with permission of the IEEE. Such permission of the IEEE does not in any way imply IEEE endorsement of any of Helsinki University of Technology's products or services. Internal or personal use of this material is permitted. However, permission to reprint/republish this material for advertising or promotional purposes or for creating new collective works for resale or redistribution must be obtained from the IEEE by writing to pubs-permissions@ieee.org.

By choosing to view this document, you agree to all provisions of the copyright laws protecting it. 
To appear in IEEE Transaction on Systems, Man, and Cybernetics - Part A: Systems and Humans.

Reprinted with the permission of IEEE.

\title{
Team Optimal Signaling Strategies in Air Combat
}

\author{
Kai Virtanen, Raimo P. Hämäläinen, Member, IEEE, and Ville Mattila
}

Abstract - Modern fighter aircraft are equipped with a data link system for relaying encrypted messages containing information on the states of friendly or hostile aircraft within a team of friendly aircraft. During a many-versus-many air combat, only the most important and essential information must be transmitted in order to achieve the best possible situation awareness of pilots. In this paper, we introduce a prioritization approach for solving the team optimal signaling strategy referring to the best sequence of relayed information with respect to the overall goals of the friendly pilots. The approach is based on a value function that captures the preferences of the pilots and prioritizes the existing information. Uncertainty about the states of the aircraft as well as about incomplete preference statements is incorporated into the prioritization by utilizing interval analysis. We also describe a software that is designed for simulating the evolution of team members' information and trajectories in an air combat game. The prioritization approach is illustrated by numerical examples.

Index Terms - Aircraft expert systems, communication systems, decision analysis, incomplete information, military information systems, team decision-making, uncertainty

\section{INTRODUCTION}

In air combat, pilots have to make frequent decisions about, e.g., maneuvering, based on information received from different sources. They evaluate the states of both friendly and hostile aircraft by visual detection, radio communications, or by positioning them with a radar. The pilot can also obtain information from a data link system (e.g., [11], [25], [28]). It offers a communications channel between the aircraft that are members of a signaling network. The data link system automatically relays encrypted messages and provides a reliable way to transmit information under electronic countermeasures generated by a hostile force. The system is developed to enable the pilots to share information about the combat state and to enhance their situation awareness. Hence, it helps the situation assessment of the pilots and supports collaborative decision making.

Data link messages contain state information about an aircraft, friendly or hostile. An example air combat situation in which such a message related to a hostile aircraft increases considerably the understanding of the pilot about the combat state is shown in Fig. 1. The pilots must always know the states of the other friendly aircraft because each aircraft seen on the radar display is assumed to be hostile unless it is not identified as a friendly one. Thus, the data link system must also send the state information on friendly aircraft, see Fig. 2. The system under consideration does not contain a confirmation mechanism that would inform the members of the signaling network about the success of transmission of single messages. For this reason, messages about important aircraft should be retransmitted more frequently than messages containing information about less important aircraft.

The authors are with the Systems Analysis Laboratory, Helsinki University of Technology (HUT), P.O.Box 1100, FIN-02015 HUT, Finland, www.sal.hut.fi (e-mails: kai.virtanen@hut.fi, raimo@hut.fi, ville.a.mattila@hut.fi). 


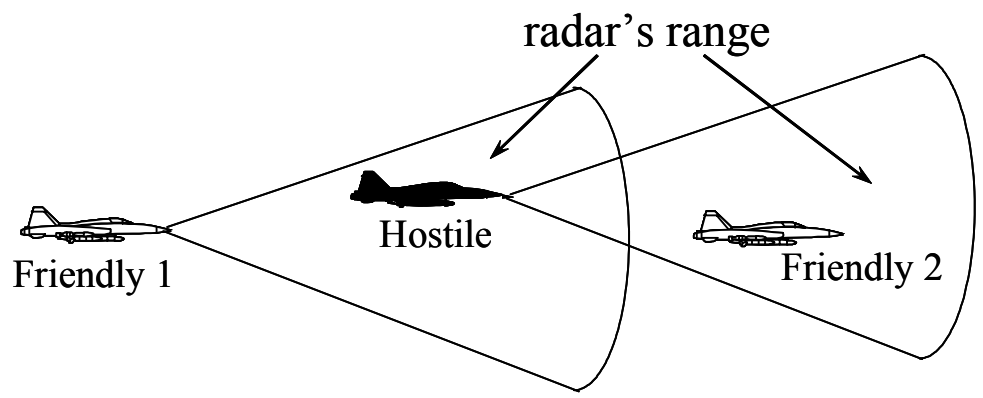

Fig. 1: Friendly 1 must transmit a data link message about the hostile aircraft in order to make Friendly 2 aware of the hostile one.

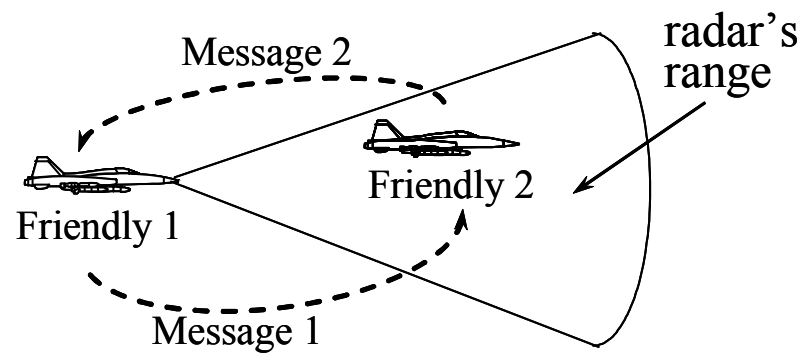

Fig. 2: Message 1 is needed to make Friendly 2 aware of Friendly 1 . Message 2 informs Friendly 1 that Friendly 2 is a friendly aircraft.

The data link system cannot relay all the existing information because its transmission capacity is limited. Hence, the most important and essential information with respect to the overall goals of the friendly team should be identified by prioritizing the dynamically varying importance of the aircraft. This creates the signaling problem whose solution gives the team optimal signaling strategy that refers to the best sequence of state information included in successive data link messages transmitted by the friendly aircraft. In this paper, we introduce a prioritization approach for solving such a strategy during a many-versus-many air combat.

The signaling problem can be considered a dynamic team decision problem under conditions of uncertainty [2], [6], [10]. In such a problem, a team is defined as a group of team members who aim at achieving a common goal by acting in a dynamic, hostile, and uncertain environment. At each transmission instant of the signaling problem, one team member, i.e., the transmitting friendly aircraft, prioritizes and selects the contents of a message to be transmitted. Only those hostile aircraft that are seen by the radar of the transmitting aircraft are permitted in the prioritization. The transmitting aircraft can also send information on its own state. The objective function of the signaling problem should represent the common goal of the pilots as accurately as possible. It must associate a high priority with the aircraft whose current state information leads to the highest improvement in the situation awareness of the friendly pilots. The aircraft are prioritized based on information received from radar observations as well as from earlier messages provided by the data link system. The available information is uncertain because radar errors and old data link information does not coincide with the present combat state.

The early literature on team decision theory focuses on linear-quadratic models (e.g., [1], [2], [10], [29]). In these models, a linear difference or differential equation represents the evolution of the decision environment in time. A quadratic objective function describes the goal of a team and the probability theory is used for modeling uncertainties. Unfortunately, these approaches cannot be applied to the signaling problem. In practice, the quadratic objective function is often a limited and unrealistic representation for the pilots' goals. Team decision models in which the objective function is more complex and uncertainty is 
represented by a probability measure have proven to be computationally intractable. On the other hand, the impact of the transmitted information on the maneuvers of the pilots and, further, the states of their aircraft are not known certainly. Thus, it is impossible to construct a set of difference or differential equations representing the dynamics of the combat in which control variables refer to the transmitted information and state variables to the state of the combat, respectively.

Due to the lack of equations describing the evolution of the combat state as a function of the transmitted information, the dynamic team decision problem is now replaced with a set of static team problems [3] representing the signaling problem at each single transmission instant. In our prioritization approach, the main building block of the objective function of these signaling problems is a value function (e.g., [14], [15]). It is a widely utilized method from decision analysis for analyzing multicriteria decision problems. Hereafter in this paper, the term value function refers to the objective function of the signaling problem.

The value function models the preferences of the pilots and evaluates the individual importance of a hostile aircraft from the viewpoint of each member of the friendly team. In practice, the value function maps the momentary combat state onto the importance measure. In addition, the importance of a given aircraft is influenced by the elapsed time since the previous transmission instant of the message about this aircraft. The time factor enables the data link system to retransmit information about a particular aircraft although it would have low importance calculated based on the combat state only. The overall importance index of the hostile aircraft is determined by aggregating the individual one-on-one importances. Because the transmitting aircraft can also send its own state information, an importance index for it is needed. This index is constructed by averaging the importance indices associated with earlier transmitted messages. The final selection of the contents of the data link messages is made based on the importance indices of the hostile and transmitting aircraft.

We use an interval approach instead of the probability theory for modeling the underlying uncertainty of the signaling problem. Therefore, resulting team optimal signaling strategies can be determined although the value function is nonquadratic. Interval analysis has previously been applied to incorporate uncertainties in decision models [21]-[23]. It is also used in group decision making to aggregate the preferences of all group members in a single model [12]. In the signaling problem, the state information of the aircraft is not known precisely and, in addition, incomplete preference statements can lead to imprecise parameters of the value function. The state variables and the parameters are now allowed to vary within given ranges. One-on-one importance intervals are calculated with nonlinear programming (e.g., [5]) by maximizing and minimizing the value function subject to the feasible ranges. Then, the prioritization approach gives an interval for the overall importance index of each hostile aircraft by aggregating the importance intervals. This allows the ranking of the state information of the aircraft in an order of importance. The intervals can also be utilized in sensitivity analysis [17] in which the impacts of the different factors affecting the prioritization can be found out.

Computer support has been used successfully for bringing decision theoretic methods into practice. Decision models based on value functions and intervals can be structured, solved, and validated with the help of existing decision support software (e.g., [13], [19]). These software have been developed for modeling and analyzing single decision problems containing static information defined and given by the user of the software. They are not suitable for tackling the signaling problem in which the available information varies in time. The presented prioritization approach is implemented in a Matlab-based [18] software called Information Prioritization in Air Combat (IPAC). It simulates and visualizes the signaling and flight paths of the involved aircraft in a multiplayer air combat game. IPAC also offers features for eliciting preferences from pilots into the value function. The user interface of IPAC resembles a radar screen and thus different signaling strategies can be compared and validated on a realistic display. 
The paper is organized as follows. Section II formulates the signaling problem which emerges from the prioritization of state information included in data link messages during an air combat. Section III introduces the prioritization approach utilizing perfect information only and describes the structure of the value function used for modeling the preferences of pilots. In Section IV, uncertainties about the states of the aircraft as well as about preference statements are incorporated into the prioritization by applying interval analysis. The IPAC simulation software is described in Section V. In Section VI, the presented prioritization approach is illustrated and compared to a commonly used prioritization measure by simulation examples. In addition, an example sensitivity analysis is carried out with the IPAC software. Finally, concluding remarks appear in Section VII.

\section{SIGNALING PROBLEM}

We consider an M-on-N air combat where two groups of aircraft, the friendly team and the adversary team, fight against each other. The friendly team, denoted by $\mathbf{T M}$, includes $\mathbf{T M} i \in \mathbf{T} \mathbf{M}=\{\mathbf{T} \mathbf{M} 1, \ldots, \mathbf{T} \mathbf{M} M\}$ members and the adversary team $\mathbf{O P} \mathbf{O P} j \in \mathbf{O P}=\{\mathbf{O P} 1, \ldots, \mathbf{O P} N\}$ opponents, respectively. During the combat, each member of the friendly team utilizes a data link system for transmitting information to the other friendly members through a signaling network. In the network, the members relay messages successively at discrete time instants $\mathrm{t}=0,1,2, \ldots, \mathrm{T}$, where $\mathrm{T}$ is the duration of the combat. The transmission turn circulates among the team members according to a prespecified order. Let the number of the turns be $\mathrm{K}$ and $\mathrm{i}_{\mathrm{k}}=(\mathrm{i}-1)+(\mathrm{k}-1) \mathrm{M}, \mathrm{k}=1,2, \ldots, \mathrm{K}$, be the transmission instants of $\mathbf{T M} i$, respectively. The last message is sent by $\mathbf{T M} M$ at $\mathrm{M}_{\mathrm{K}}=\mathrm{T}$.

The state of each team member's aircraft at time $\mathrm{t}$ is described by the state vector $\mathbf{T} \mathbf{M} i{ }^{\mathrm{t}}=\left[\mathrm{x} i{ }^{\mathrm{t}} \mathrm{y} i{ }^{\mathrm{t}} \mathrm{h} i{ }^{\mathrm{t}} \mathrm{v} i_{\mathrm{x}}{ }^{\mathrm{t}} \mathrm{v} i_{\mathrm{y}}{ }^{\mathrm{t}}\right.$ $\left.v i_{\mathrm{h}}{ }^{\mathrm{t}} \mathrm{a} i_{\mathrm{x}}{ }^{\mathrm{t}} \mathrm{a} i_{\mathrm{y}}{ }^{\mathrm{t}} \mathrm{a} i_{\mathrm{h}}{ }^{\mathrm{t}}\right]^{\mathrm{T}}$ where the variables refer to the $\mathrm{x}$-coordinate, the y-coordinate, altitude, the $\mathrm{x}-, \mathrm{y}-$ and altitude components of velocity and the $\mathrm{x}-, \mathrm{y}$ - and altitude components of acceleration, respectively. The coordinate frame represents flat Earth. If the combat area is large, $\mathrm{x}$ - and y-coordinates can be replaced with latitude and longitude. $\mathbf{O P} j^{\mathrm{t}}$ denotes the state vector of $\mathbf{O P} j$ and it contains the same variables as the team member's state vector.

The team members are not aware of the exact states of the aircraft because of errors in radar measurements as well as of nonupdated information that has been received from earlier data link messages. Each state

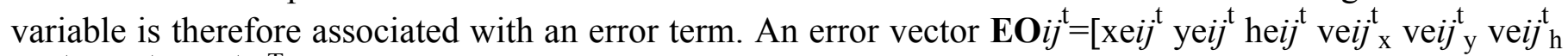
$\operatorname{ae}_{i j}{ }_{\mathrm{x}}^{\mathrm{t}}$ aeij ${ }_{\mathrm{y}}^{\mathrm{t}}$ aeij $\left.{ }_{\mathrm{h}}^{\mathrm{t}}\right]^{\mathrm{T}}$ reflects the uncertainty in the understanding of TM $i$ about the state of $\mathbf{O P} j$ at time $\mathrm{t}$. Here, the variables of the error vector refer to the corresponding state variables, and the true state belongs to the interval $\left[\mathbf{O P} j^{\mathrm{t}}-\mathbf{E} \mathbf{O} i j^{\mathrm{t}}, \mathbf{O P} j^{\mathrm{t}}+\mathbf{E O} i{ }^{\mathrm{t}}{ }^{\mathrm{t}}\right]$. The error vector $\mathbf{E T} i l^{\mathrm{t}}$ infers the uncertain understanding of $\mathbf{T M} i$ about the state of $\mathbf{T M} l$ at time t. The accuracy of information included in a message received from the data link some time ago decreases with time because the states of the aircraft evolve dynamically. Hence, the error terms of a particular aircraft increase in time. The team members will ignore state information older than a maximum memory time $t_{\max }$.

A data link message can contain state information about an opponent tracked by the radar of the transmitting team member. Denote the set of the opponents tracked by $\mathbf{T M} l$ at time $\mathrm{t}$ as $\mathbf{T R} l^{\mathrm{t}} \subseteq \mathbf{O P}$ and let $\mathbf{I} l^{\mathrm{t}}=\left\{j \mid \mathbf{O P} j \in \mathbf{T R} l^{\mathrm{t}}\right\}$ be the set of the corresponding indices. The transmitting team member can also relay a message about the state of its own aircraft. If $\mathbf{T M} l$ is relaying at time t, the set of feasible aircraft whose state information can be included in the message is $\mathbf{M A} l^{\mathrm{t}}=\{\mathbf{T M} l\} \cup \mathbf{T R} l^{\mathrm{t}}$. Then, $\mathbf{T M} l$ has to rank the feasible aircraft that belong to the set $\mathbf{M A} l^{\mathrm{t}}$ and select the most important aircraft with respect to the 
common goal of the team. Denote the set of the team members known by TM $l$ at time $\mathrm{t}$ as $\mathbf{T K} l^{\mathrm{t}} \subseteq \mathbf{T M}$ and let $\mathbf{I K} l^{\mathrm{t}}=\left\{i \mid \mathbf{T M} i \in \mathbf{T K} l^{\mathrm{t}}\right\}$ be the set of the corresponding indices. The transmitting team member $\mathbf{T M} l$ does not belong to the set $\mathbf{T K} l^{\mathrm{t}}$. The $\mathbf{T M} l$ 's previous transmission or receiving time of a message on $\mathbf{O P} j$ is denoted by $\mathrm{t} l j$ and the $\mathbf{T M} l$ 's last transmission time of the own state information by $\mathrm{t} l$, respectively.

During the combat, each team member receives information from data link messages and radar observations which determines the information set available specifically to the particular team member. The members use this data for prioritizing the aircraft. At time $t, \mathbf{I O} l^{\mathrm{t}}$ denotes the information set of TM $l$ and it consists of

- state vector of $\mathbf{T M} l^{\mathrm{t}}$

- state $\mathbf{T M} i{ }^{\mathrm{t}}$ and error $\mathbf{E T} l i^{\mathrm{t}}$ vectors of the team members which are tracked by the radar of $\mathbf{T M} l$ at time $\mathrm{t}$

- state $\mathbf{T M} i^{\mathrm{t}}$ and error $\mathbf{E T} l i^{\mathrm{t}}$ vectors of the team members whose state information has been received by $\mathbf{T M} l$ from the data link messages within the maximum memory time $t_{\max }$

- state $\mathbf{O P} j^{\mathrm{t}}$ and error $\mathbf{E O} l j^{\mathrm{t}}$ vectors of the opponents which are tracked by the radar of $\mathbf{T M} l$ at time $\mathrm{t}$

- state $\mathbf{O P}{ }^{\mathrm{t}}$ and error $\mathbf{E O} l j^{\mathrm{t}}$ vectors of the opponents whose state information has been received by $\mathbf{T M} l$ from the data link messages within the maximum memory time $t_{\max }$

- most recent transmission time of own state information $t l$

- most recent transmission or receiving times of the information about the known opponents $t j j$

Each team member's decisions about the contents of transmitted messages have an effect on the outcome of the team's common goal as well as on the information received by the other team members. Hence, the signaling problem can be formulated as a dynamic team decision problem

$$
\max _{\substack{\mathrm{u}_{\mathrm{i} k} \\ \mathrm{k}=1, \ldots, \mathrm{K} \\ \mathrm{i}=1, \ldots, \mathrm{M}}} \sum_{\mathrm{k}=1}^{\mathrm{K}} \sum_{\mathrm{i}=1}^{\mathrm{M}} \widetilde{\mathrm{J}}\left(\mathrm{u}^{\mathrm{i}_{\mathrm{k}}}, \mathbf{I O} i^{\mathrm{i}_{\mathrm{k}}}\right)
$$

subject to

$$
\begin{gathered}
\mathrm{u}^{\mathrm{i}_{\mathrm{k}}}=\mathrm{u}^{\mathrm{i}_{\mathrm{k}}}\left(\mathbf{I O} i^{\mathrm{i}_{\mathrm{k}}}\right) \in \mathbf{M A} i^{\mathrm{i}_{\mathrm{k}}}, \\
\mathbf{I O} i^{{ }^{\mathrm{k}}{ }_{\mathrm{k}}} \text { depends on } \mathbf{I O} i^{{ }^{\mathrm{k}}-1} \text { and } \mathrm{u}^{\mathrm{i}_{\mathrm{k}}-1}, \\
\mathrm{i}=1, \ldots, \mathbf{M}, \mathrm{k}=1, \ldots, \mathrm{K} .
\end{gathered}
$$

Here $\widetilde{\mathbf{J}}$ is the value function that describes the common goal of the friendly team and $\mathrm{u}^{\mathrm{i}_{\mathrm{k}}}=\mathrm{u}^{\mathrm{i}_{\mathrm{k}}}\left(\mathbf{I O} i^{\mathrm{i}_{\mathrm{k}}}\right)$ is the decision variable referring to the aircraft whose state information is transmitted at time $i_{k}$. Messages relayed earlier have an effect on the future maneuvers of the team members and the opponents. Therefore, the messages also contribute to the upcoming combat states and information sets. This dynamics in the information structure of the combat is represented by the dependency (3). If this dependency is expressed explicitly, the team optimal signaling strategy over the total duration of the combat could be determined by solving (1)-(3) with dynamic programming [4]. In practice, the realistic formulation of (3) is an impossible task because one does not know how the transmitted information affects the flight tactics and strategies of both friendly and hostile pilots which determine the evolution of the combat state.

In this paper, the signaling problem is formulated such that the team members utilize only the momentary information set and do not anticipate future actions of the other friendly members and the opponents. The problem (1)-(3) is divided into the set of static team decision problems

$$
\max _{\mathrm{u}^{\mathrm{i}_{\mathrm{k}}}} \widetilde{\mathrm{J}}\left(\mathrm{u}^{\mathrm{i}_{\mathrm{k}}}, \mathbf{I O} i^{\mathrm{i}_{\mathrm{k}}}\right)
$$

subject to 


$$
\begin{gathered}
\mathrm{u}^{\mathrm{i}_{\mathrm{k}}}=\mathrm{u}^{\mathrm{i}_{\mathrm{k}}}\left(\mathbf{I O} i^{\mathrm{i}_{\mathrm{k}}}\right) \in \mathbf{M A} i^{\mathrm{i}_{\mathrm{k}}}, \\
\text { given } \mathbf{I O} i^{\mathrm{i}_{\mathrm{k}}}, \\
\mathrm{i}=1, \ldots, \mathrm{M}, \mathrm{k}=1, \ldots, \mathrm{K},
\end{gathered}
$$

which are illustrated in Fig. 3. The solutions of the problems above give the team optimal signaling strategy for the team members. Now, the prioritization of the state information included in the message to be transmitted at a particular transmission instant is based only on the known information set because the dynamics of the combat's information structure given by (3) is omitted.

\section{Decision at $1_{k}$}

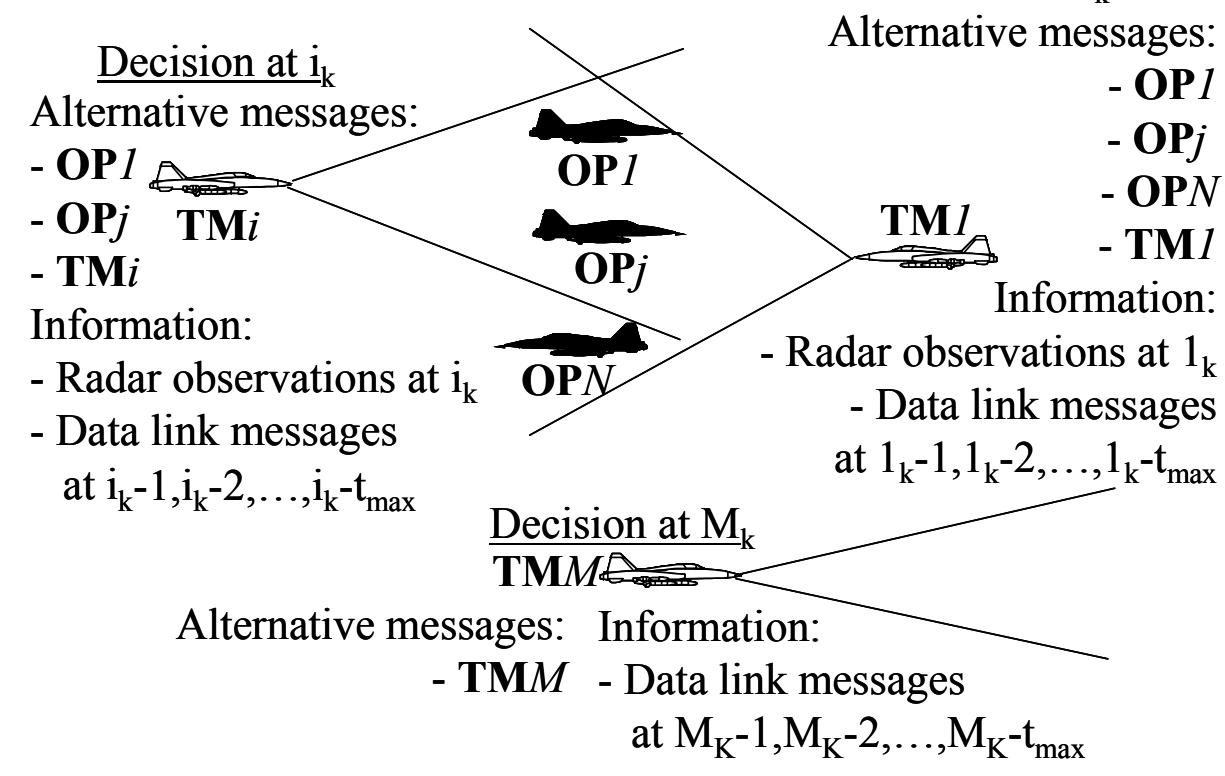

Fig. 3: The signaling problem.

\section{PRIORITIZATION UNDER CERTAINTY}

The value function $\widetilde{J}$ in (4) modeling the goals and preferences of the friendly team is the most essential part in the signaling problems (4)-(6). It ranks the aircraft such that information about the most important aircraft is relayed at each transmission instant. In practice, the function must map the current information set onto a measure representing the priorities of the aircraft. Hereafter, this measure is called the importance index. For each opponent, the index is obtained by aggregating one-on-one importances that measure the individual importance of an opponent with respect to one known team member, see Fig. 4. The importance index describes the overall importance of an opponent with respect to the whole friendly team, see Fig. 5. The importance index is also calculated for the transmitting aircraft so that it can be compared to the opponents. In this section, the determination of the importance index and a procedure for solving team optimal signaling strategies are described by omitting the underlying uncertainty of the signaling problem.

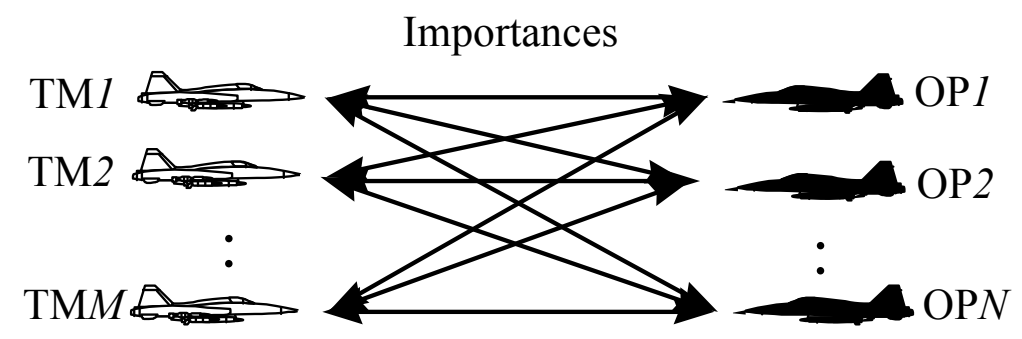

Fig. 4: Importance is the importance of an individual opponent with respect to an individual team member. 


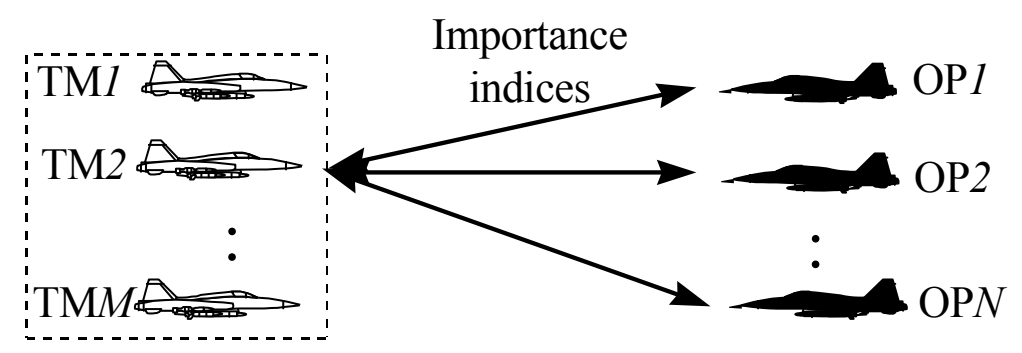

Fig. 5: Importance index is the importance of an individual opponent with respect to the whole friendly team.

\section{A. Importance Index}

The one-on-one importances are calculated using a decision theoretical value function [14], [15]. In general, such a function provides a way to quantitatively evaluate the outcomes of decision alternatives by utilizing a set of attributes. In the signaling problem, the attributes of the value function represent the relative combat state between a team member and an opponent as well as elapsed time from the previous transmission related to a particular aircraft. Each attribute is mapped into a value scale with a single attribute value function. The single values are combined with an aggregation function giving one-on-one importances that are used for determining the final importance index for each opponent.

Consider first the combat state attributes that are included in the attribute vector $\mathbf{C S} i{ }^{\mathrm{t}}{ }^{\text {describing }}$ the combat state between a team member TM $i$ and an opponent $\mathbf{O P} j$ at time t. Naturally, the attribute vector can only be calculated for the aircraft whose states are in the information set of the transmitting team member. The attributes are functions of the state variables that are available in the data link system, i.e.,

$$
\begin{gathered}
\mathbf{C S} i j^{\mathrm{t}}=\left[\alpha i j^{\mathrm{t}} \beta i j^{\mathrm{t}^{\mathrm{t}}} \mathrm{d} i j^{\mathrm{t}} \Delta \mathrm{d} i j^{\mathrm{t}} \Delta v i j^{\mathrm{t}} \Delta \mathrm{h} i j^{\mathrm{t}} \mathrm{a} i j^{\mathrm{t}}\right]^{\mathrm{T}}= \\
{\left[\mathrm{f}_{\alpha}(\bullet) \mathrm{f}_{\beta}(\bullet) \mathrm{f}_{\mathrm{d}}(\bullet) \mathrm{f}_{\Delta \mathrm{d}}(\bullet) \mathrm{f}_{\Delta \mathrm{v}}(\bullet) \mathrm{f}_{\Delta \mathrm{h}}(\bullet) \mathrm{f}_{\mathrm{a}}(\bullet)\right]^{\mathrm{T}}=\mathbf{f}\left(\mathbf{T M}{ }^{\mathrm{t}}, \mathbf{O P} j^{\mathrm{t}}\right) .}
\end{gathered}
$$

The first components of the attribute vector, see Fig. 6, are the angle between the line of sight (LOS) and the velocity vector of $\mathbf{T M} i$, the deviation angle $\alpha i j^{\mathrm{t}}$,

$$
\arccos \left(\frac{\mathrm{f}_{\alpha}\left(\mathbf{T M} i^{\mathrm{t}}, \mathbf{O P} j^{\mathrm{t}}\right)=}{\left.\sqrt{\left.\left.\left(\mathrm{x} j^{\mathrm{t}}-\mathrm{x} i^{\mathrm{t}}\right) \mathrm{v} i_{\mathrm{x}}^{\mathrm{t}}+\left(\mathrm{y} j^{\mathrm{t}}-\mathrm{x} i^{\mathrm{t}}\right)^{2}+\left(\mathrm{y} i^{\mathrm{t}}\right) \mathrm{v} i_{\mathrm{y}}^{\mathrm{t}}-\mathrm{y} i^{\mathrm{t}}\right)^{2}+\left(\mathrm{h} j^{\mathrm{t}}-\mathrm{h}-\mathrm{h} i^{\mathrm{t}}\right)^{2}\right) v i_{\mathrm{h}}^{\mathrm{t}} \sqrt{\mathrm{v} i_{\mathrm{x}}^{\mathrm{t}^{2}}+\mathrm{v} i_{\mathrm{y}}^{\mathrm{t}^{2}}+\mathrm{v} i_{\mathrm{h}}^{\mathrm{t}^{2}}}}\right),}\right.
$$

the angle between LOS and the velocity vector of $\mathbf{O P} j$, the angle off $\beta i j{ }^{\text {t }}$,

$$
\arccos \left(\frac{\mathrm{f}_{\beta}\left(\mathbf{T M} i^{\mathrm{t}}, \mathbf{O P} j^{\mathrm{t}}\right)=}{\sqrt{\left.\left(\mathrm{x} j^{\mathrm{t}}-\mathrm{x} j^{\mathrm{t}}-\mathrm{x} i^{\mathrm{t}}\right) \mathrm{vj}_{\mathrm{x}}^{\mathrm{t}}+\left(\mathrm{y} j^{\mathrm{t}}-\mathrm{y} i^{\mathrm{t}}\right) \mathrm{v} j_{\mathrm{y}}^{\mathrm{t}}+\left(\mathrm{h} j^{\mathrm{t}}-\mathrm{y} i^{\mathrm{t}}\right)^{2}+\left(\mathrm{h} j^{\mathrm{t}}\right) \mathrm{v} j_{\mathrm{h}}^{\mathrm{t}}-\mathrm{h} i^{\mathrm{t}}\right)^{2}} \sqrt{\mathrm{v}_{\mathrm{x}}^{\mathrm{t}^{2}}+\mathrm{vj}_{\mathrm{y}}^{\mathrm{t}^{2}}+\mathrm{v} j_{\mathrm{h}}^{\mathrm{t}^{2}}}}\right),
$$

the distance between the players, $\mathrm{d} i j^{\mathrm{t}}$,

$$
\mathrm{f}_{\mathrm{d}}\left(\mathbf{T M} i^{\mathrm{t}}, \mathbf{O P} j^{\mathrm{t}}\right)=\sqrt{\left(\mathrm{x} j^{\mathrm{t}}-\mathrm{x} i^{\mathrm{t}}\right)^{2}+\left(\mathrm{y} j^{\mathrm{t}}-\mathrm{y} i^{\mathrm{t}}\right)^{2}+\left(\mathrm{h} j^{\mathrm{t}}-\mathrm{h} i^{\mathrm{t}}\right)^{2}}
$$

and the approaching velocity between the players, $\Delta \mathrm{d} i j^{\mathrm{t}}$, that is the negative time derivative of the distance

$$
\begin{gathered}
\mathrm{f}_{\Delta \mathrm{d}}\left(\mathbf{T M} i^{\mathrm{t}}, \mathbf{O P} j^{\mathrm{t}}\right)= \\
-\frac{\left(\mathrm{x} j^{\mathrm{t}}-\mathrm{x} i^{\mathrm{t}}\right)\left(\mathrm{v} j_{\mathrm{x}}^{\mathrm{t}}-\mathrm{v} i_{\mathrm{x}}^{\mathrm{t}}\right)+\left(\mathrm{y} j^{\mathrm{t}}-\mathrm{y} i^{\mathrm{t}}\right)\left(\mathrm{v} j_{\mathrm{y}}^{\mathrm{t}}-\mathrm{v} i_{\mathrm{y}}^{\mathrm{t}}\right)+\left(\mathrm{h} j^{\mathrm{t}}-\mathrm{h} i^{\mathrm{t}}\right)\left(\mathrm{v} j_{\mathrm{h}}^{\mathrm{t}}-\mathrm{v} i_{\mathrm{h}}^{\mathrm{t}}\right) .}{\mathrm{d} i j^{\mathrm{t}}} .
\end{gathered}
$$


Note that $\Delta \mathrm{d} i j^{\mathrm{t}}$ is positive when the players are approaching each other. The effect of the players' energy is described by the difference between the squares of the velocities, $\Delta v i j^{t}$,

$$
\mathrm{f}_{\Delta \mathrm{v}}\left(\mathbf{T M} i^{\mathrm{t}}, \mathbf{O P} j^{\mathrm{t}}\right)=\left(\mathrm{v} j_{\mathrm{x}}^{\mathrm{t}}\right)^{2}+\left(\mathrm{v} j_{\mathrm{y}}^{\mathrm{t}}\right)^{2}+\left(\mathrm{v} j_{\mathrm{h}}^{\mathrm{t}}\right)^{2}-\left(\mathrm{v} i_{\mathrm{x}}^{\mathrm{t}}\right)^{2}-\left(\mathrm{v} i_{\mathrm{y}}^{\mathrm{t}}\right)^{2}-\left(\mathrm{v} i_{\mathrm{h}}^{\mathrm{t}}\right)^{2}
$$

and the difference between the altitudes of the players, $\Delta \mathrm{h} i j^{\mathrm{t}}$,

$$
\mathrm{f}_{\Delta \mathrm{h}}\left(\mathbf{T M} i^{\mathrm{t}}, \mathbf{O P} j^{\mathrm{t}}\right)=\mathrm{h} j^{\mathrm{t}}-\mathrm{h} i^{\mathrm{t}} \text {. }
$$

A possible change in the maneuvering of $\mathbf{O P} j$ is indicated by the acceleration, $a i j$,

$$
\mathrm{f}_{\mathrm{a}}\left(\mathbf{T M} i^{\mathrm{t}}, \mathbf{O P} j^{\mathrm{t}}\right)=\sqrt{\mathrm{aj}_{\mathrm{x}}^{\mathrm{t}^{2}}+\mathrm{a} j_{\mathrm{y}}^{\mathrm{t}^{2}}+\mathrm{a} j_{\mathrm{h}}^{\mathrm{t}^{2}}} \text {. }
$$

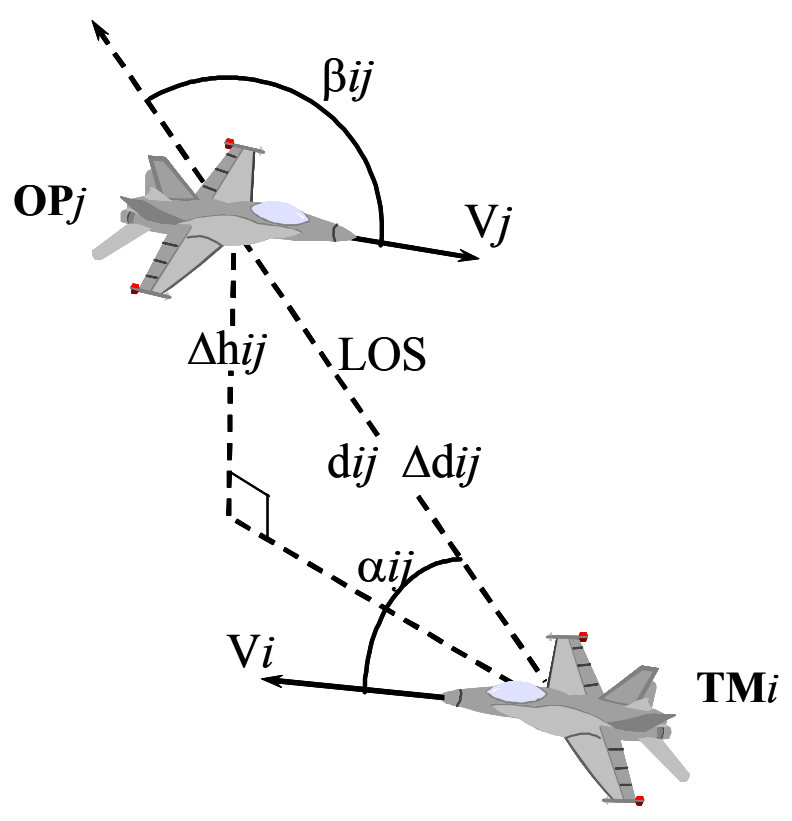

Fig. 6: The attributes of the value function.

The value function related to the combat state is formed by aggregating single attribute value functions $\mathrm{v}_{\alpha}$, $\mathrm{v}_{\beta}, \mathrm{v}_{\mathrm{d}}, \mathrm{v}_{\Delta \mathrm{d}}, \mathrm{v}_{\Delta \mathrm{v}}, \mathrm{v}_{\Delta \mathrm{h}}$, and $\mathrm{v}_{\mathrm{a}}$ that are scaled such that the best level of an attribute is assigned the value 1 and the worst 0 , respectively. The simplest form of aggregation is additive, i.e., a linear combination of the single attribute value functions [14], [15]. It is valid only if the attributes are preferentially independent [14], [15]. Two attributes are preferentially independent, if the preference order for the level of one attribute does not depend on the level of the other attribute. In the signaling problem, the attributes do not satisfy this criterion. For example, let us examine the deviation angle $\alpha$ and the angle off $\beta$, and assume that the distance between the aircraft is less than the maximum reach of the radar. If $\alpha$ is within the viewing angle of the radar of the team member, we are indifferent to the level of $\beta$ because the opponent is in the radar beam of the team member regardless of $\beta$. On the other hand, if $\alpha$ is outside of the viewing angle, large values of $\beta$ are preferred because then the opponent can see the team member which is impossible when $\beta$ gets close to zero.

When the attributes are preferentially dependent, a multiplicative value function is appropriate (e.g., [15]). It is composed by adding product terms of single attribute value functions to the additive model. The importance of $\mathbf{O P} j$ with respect to $\mathbf{T M} i$ is calculated with a multiplicative value function of the form 


$$
\begin{gathered}
\mathrm{v}\left(\mathbf{C S} i j^{\mathrm{t}} ; \mathbf{w}\right)=\mathbf{w}^{\mathrm{T}} \mathbf{v}\left(\mathbf{C S} i j^{\mathrm{t}}\right)= \\
\mathrm{w}_{\alpha} \mathrm{v}_{\alpha}\left(\alpha i j^{\mathrm{t}}\right)+\mathrm{w}_{\beta} \mathrm{v}_{\beta}\left(\beta i j^{\mathrm{t}}\right)+\mathrm{w}_{\mathrm{d}} \mathrm{v}_{\mathrm{d}}\left(\mathrm{d} i j^{\mathrm{t}}\right)+\mathrm{w}_{\Delta \mathrm{d}} \mathrm{v}_{\Delta \mathrm{d}}\left(\Delta \mathrm{d} i j^{\mathrm{t}}\right)+\mathrm{w}_{\Delta \mathrm{v}} \mathrm{v}_{\Delta \mathrm{v}}\left(\Delta \mathrm{v} i j^{\mathrm{t}}\right)+ \\
\mathrm{w}_{\Delta \mathrm{h}} \mathrm{v}_{\Delta \mathrm{h}}\left(\Delta \mathrm{h} i j^{\mathrm{t}}\right)+\mathrm{w}_{\mathrm{a}} \mathrm{v}_{\mathrm{a}}\left(\mathrm{a} i j^{\mathrm{t}}\right)+\mathrm{w}_{\alpha \mathrm{d}} \mathrm{v}_{\alpha}\left(\alpha i j^{\mathrm{t}}\right) \mathrm{v}_{\mathrm{d}}\left(\mathrm{d} i j^{\mathrm{t}}\right)+\mathrm{w}_{\alpha \Delta \mathrm{d}} \mathrm{v}_{\alpha}\left(\alpha i j^{\mathrm{t}}\right) \mathrm{v}_{\Delta \mathrm{d}}\left(\Delta \mathrm{d} i j^{\mathrm{t}}\right)+ \\
\mathrm{w}_{\alpha \mathrm{a}} \mathrm{v}_{\alpha}\left(\alpha i j^{\mathrm{t}}\right) \mathrm{v}_{\mathrm{a}}\left(\mathrm{a} i j^{\mathrm{t}}\right)+\mathrm{w}_{\beta \mathrm{d}} \mathrm{v}_{\beta}\left(\beta i j^{\mathrm{t}}\right) \mathrm{v}_{\mathrm{d}}\left(\mathrm{d} i j^{\mathrm{t}}\right)+\mathrm{w}_{\beta \Delta \mathrm{d}} \mathrm{v}_{\beta}\left(\beta i j^{\mathrm{t}}\right) \mathrm{v}_{\Delta \mathrm{d}}\left(\Delta \mathrm{d} i j^{\mathrm{t}}\right)+ \\
\mathrm{w}_{\alpha \beta \mathrm{d}} \mathrm{v}_{\alpha}\left(\alpha i j^{\mathrm{t}}\right) \mathrm{v}_{\beta}\left(\beta i j^{\mathrm{t}}\right) \mathrm{v}_{\mathrm{d}}\left(\mathrm{d} i j^{\mathrm{t}}\right)+\mathrm{w}_{\alpha \beta \Delta \mathrm{d}} \mathrm{v}_{\alpha}\left(\alpha i j^{\mathrm{t}}\right) \mathrm{v}_{\beta}\left(\beta i j^{\mathrm{t}}\right) \mathrm{v}_{\Delta \mathrm{d}}\left(\Delta \mathrm{d} i j^{\mathrm{t}}\right),
\end{gathered}
$$

where the column vector $\mathbf{V}$ contains the single attribute value functions as well as the product terms. The relative weights w. are given in the column vector $\mathbf{w}$ and sum up to one, i.e., $\|\mathbf{w}\|_{1}=1$. Thus, the importance resulting from the combat state is a real number between zero and one.

The interpretation of the weights in a multiplicative value function is not as straightforward as in additive value models. However, they can still be considered to describe the relative importance of the single terms. These weights as well as the shapes of the single attribute value functions reflect personal preferences and goals of pilots. Decision analysis offers a number of methods and tools for eliciting these factors from the pilots (e.g., [13]-[15], [19]). Preference elicitation techniques available in the IPAC software are discussed in Section V. Illustrative single attribute functions and weights are described in Section VI.

The product terms in (15) represent different preferential dependencies of the attributes. The distance and the deviation angle specify whether the opponent can be observed by the radar and thus the product term $\mathrm{v}_{\alpha} \mathrm{V}_{\mathrm{d}}$ is added into (15). The range of the opponent's radar is taken into account by the term $\mathrm{v}_{\beta} \mathrm{V}_{\mathrm{d}}$. The term $\mathrm{V}_{\alpha} \mathrm{V}_{\Delta \mathrm{d}}$ allows increasing importance for an approaching opponent that cannot be seen with the radar of the team member. On the other hand, the term $v_{\beta} v_{\Delta d}$ associates an additional importance component with the opponent flying toward the team member regardless of the level of the deviation angle. If the opponent has high acceleration and it is flying behind the team member, one can conclude that the opponent is beginning an offensive maneuver. Its impact on the importance is captured by the term $\mathrm{v}_{\alpha} \mathrm{V}_{\mathrm{a}}$. The impact of the relative combat geometry on the importance is modeled by the term $\mathrm{v}_{\alpha} \mathrm{V}_{\beta} \mathrm{v}_{\mathrm{d}}$ that gives a large contribution to the importance when the opponent is flying close behind the team member and a small contribution in an opposite situation. The opponent reaching the team member's tail must have a high importance. The situation of this type is taken into account in (15) by the term $\mathrm{v}_{\alpha} \mathrm{V}_{\beta} \mathrm{v}_{\Delta \mathrm{d}}$. Energy advantage is a critical factor in an air combat and now it influences the importance by the terms $v_{\Delta v}$ and $v_{\Delta h}$.

The impact of the age of earlier transmitted information on the importance is modeled by adding a new time-dependent attribute into (15). Suppose that $\mathbf{T M} l$ is transmitting at time $t$ and it has sent or received a message about $\mathbf{O P} j$ at time $t l j$. The age of this information is $t-t l j$. The overall value function becomes

$$
\mathrm{J}_{i j}{ }^{\mathrm{t}}=\mathrm{J}\left(\mathbf{C S} i j^{\mathrm{t}}, \mathrm{t}, \mathrm{t} l j ; \mathbf{w}\right)=\mathrm{v}_{\mathrm{t}}(\mathrm{t}-\mathrm{t} l j) \mathrm{v}\left(\mathbf{C S} i j^{\mathrm{t}} ; \mathbf{w}\right),
$$

where $\mathrm{J}_{i j}{ }^{\mathrm{t}}$ is the importance of $\mathbf{O P} j$ with respect to $\mathbf{T M} i, \mathrm{v}$ is determined by (15), and $\mathrm{v}_{\mathrm{t}}$ is the single attribute value function for the time attribute. $\mathrm{v}_{\mathrm{t}}$ is increasing and maps the elapsed time into interval [0,1]. Although Eq. (15) would give low importance for an aircraft, Eq. (16) enables the data link system to send information about this particular aircraft if the previous message about it has been transmitted or received a long time ago.

Because TM $l$ is transmitting at time t, importance indices for all $\mathbf{O P} j$ that are seen by $\mathbf{T M} l$ 's radar must be solved by aggregating the one-on-one importances obtained by (16). One possibility is to calculate a weighted sum of the importances

$$
\mathrm{Jj}^{\mathrm{t}}=\sum_{\mathrm{i} \in \mathbf{I} \mathbf{K} l^{\mathrm{t}}} \mathrm{p}_{\mathrm{i}} \mathrm{J} i j^{\mathrm{t}},
$$


where $\mathrm{J}^{\mathrm{t}}$ is the importance index of $\mathbf{O P} j$ and $\mathrm{p}_{\mathbf{i}}, \sum_{\mathrm{i}=1}^{\mathrm{M}} \mathrm{p}_{\mathrm{i}}=1$, is the weight associated with TM $i$. The weights represent the team members' relative importance in air combat. The weight of the team's flight leader could be greater than the weights of the wingmen. Recall that IK $l^{t}$ refers to the set of the indices of the team members that are known by $\mathbf{T M} l$. Eq. (17) contains the one-on-one importances between the known team members and the tracked opponents only. The one-on-one importance between $\mathbf{T M} l$ and $\mathbf{O P} j$ is ignored in (17) because the transmitting team member is aware of all the opponents that are prioritized.

Eq. (17) can give ranks that are inappropriate in some combat situations. For example, one team member can be under the threat of the opponent's weapons and, at the same time, the opponent has low importance with respect to the other team members. Then, the relayed message does not necessarily inform the threatened member of the crucial opponent. An alternative approach for combining the importances is to use the maximum importance as the importance index,

$$
\mathrm{J} j^{\mathrm{t}}=\max _{\mathrm{i} \in \mathbf{I K} l^{\mathrm{t}}}\left\{\mathrm{J} i j^{\mathrm{t}}\right\} \text {. }
$$

This can be considered as the worst case aggregation, since the importance index is based only on the highest importance.

Due to the need of messages containing information about the transmitting aircraft itself, one also has to create an importance index for the team members. This index is calculated by averaging the importance indices of the earlier transmitted messages. The importance index of $\mathbf{T M} l$ at time $1_{\mathrm{k}}$ is given by

$$
\mathrm{JT}^{1_{\mathrm{k}}}=\frac{1}{\mathrm{~L}} \mathrm{v}_{\mathrm{t}}^{\mathrm{tm}}\left(1_{\mathrm{k}}-\mathrm{t} l\right) \sum_{\mathrm{m}=1}^{\mathrm{L}} \mathrm{J}^{1_{\mathrm{k}-\mathrm{m}}},
$$

where $\mathrm{t} l$ refers to the latest time instant when $\mathbf{T M} l$ has sent its own state information, $\mathrm{J}^{\mathrm{l}_{\mathrm{k}-\mathrm{m}}}$ are the importance indices associated with the messages transmitted earlier by $\mathbf{T M} l$, and L defines the number of the messages to be included in the averaging. The transmission frequency of own state information is

controlled with the shape of the increasing single attribute value function of time denoted by $v_{t}{ }^{t m}$. The moving average (19) produces an index whose magnitude is higher than those given by (17) or (18) at intervals.

\section{B. Solution of the Signaling Problem}

The required importance indices can be determined by (19) and (17) or (18). First, the most important opponent is identified by calculating the importance index for each tracked opponent and by selecting the highest one. Then, the final selection of the contents of the message is made by comparing the indices of this opponent and the transmitting team member. To summarize, the team optimal signaling strategy for the signaling problems (4)-(6) is obtained by the following procedure:

1. Set $\mathrm{l}=1$ and $\mathrm{k}=1$.

2. At $\mathrm{t}=(1-1)+(\mathrm{k}-1) \mathrm{M}$, determine the importance $\mathrm{J} i j^{\mathrm{t}} \forall i \in \mathbf{I K} l^{\mathrm{t}}, \forall j \in \mathbf{I} l^{\mathrm{t}}$ by using (16) with $\mathbf{I O} l^{\mathrm{t}}$.

3. Determine the importance index $\mathrm{J} j^{\mathrm{t}}$ for all.$j \in \mathbf{I} l^{\mathrm{t}}$ by aggregating the importances with (17) or (18).

4. Select the most important opponent based on the importance indices.

5. Select the most important aircraft by comparing the importance index of the most important opponent and the importance index of the transmitting team member $\mathbf{T M} l$ that is given by (19).

6. Transmit the data link message containing the state information of the most important aircraft.

7. If $1<\mathrm{M}$, set $\mathrm{l}=1+1$ and return to 2 . Otherwise, go to 8 .

8. If $\mathrm{k}=\mathrm{K}$, terminate. Otherwise, set $\mathrm{l}=1, \mathrm{k}=\mathrm{k}+1$ and return to 2 . 
Recall that the set $\mathbf{I} l^{\mathrm{t}}$ contains the indices of the opponents that are tracked by the transmitting team member $\mathbf{T M} l$ and $\mathbf{I O} l^{\mathrm{t}}$ denotes the information set available to $\mathbf{T M} l$ at time t.

\section{PRIORITIZATION UNDER UNCERTAINTY}

In Section III, all the state variables of the aircraft and the parameters of the value function are assumed to be accurate although we have pointed out that the prioritization is made under conditions of uncertainty. In this section, the uncertainties are incorporated into the prioritization by replacing the point-estimate weights and the attributes in (15) with intervals.

The error vectors describing the team members' uncertainty about the states of the aircraft impose intervals on the attributes. Imprecise or inconsistent preference information is taken into account by allowing an expert to give interval judgments on the weights. The use of the interval attributes and weights leads to the interval importance index measuring the importance of a single opponent with respect to the whole friendly team. This index is determined by aggregating one-on-one interval importances that describe the individual importance of an opponent from the point of view of each team member.

In the following, one-on-one interval importances are obtained by solving nonlinear optimization problems whose objective function is the overall value function constructed in Section III A. The constraints of these problems represent the feasible ranges of the attributes and the weights. Then, the resulting one-on-one interval importances are aggregated by utilizing a set of dominance concepts that give the interval importance index for each opponent. Finally, the opponents are ranked by comparing the interval importance indices with an appropriate selection rule.

\section{A. Interval Importance Index}

In the determination of interval importances, the same value function (16) is used as in the previous section but now the weights and attributes of the value function can vary within given ranges. This generates an interval for one-on-one importance. Suppose that $\mathbf{T M} l$ is the transmitting team member at time t. Denote the upper and lower bounds of the interval importance of $\mathbf{O P} j$ with respect to TM $i$ as $\overline{\mathrm{J}} \boldsymbol{i} j^{\mathrm{t}}$ and $\underline{\mathrm{J}}^{\mathrm{j}}{ }^{\mathrm{t}}$. These bounds are obtained by maximizing and minimizing the overall value function subject to the ranges of the attributes and the weights, i.e.,

$$
\overline{\mathrm{J}}_{i j}^{\mathrm{t}} / \underline{\mathrm{J} i j}^{\mathrm{t}}=\underset{\mathbf{C S}, \mathbf{w}}{\max } / \min \mathrm{J}(\mathbf{C S}, \mathrm{t}, \mathrm{t} l j ; \mathbf{w})
$$

subject to

$$
\begin{gathered}
\underline{\mathbf{C S} i j}^{\mathrm{t}} \leq \mathbf{C S} \leq \overline{\mathbf{C S}} i j^{\mathrm{t}}, \\
\underline{\mathbf{w}} \leq \mathbf{w} \leq \overline{\mathbf{w}}, \\
\|\mathbf{w}\|_{1}=1,
\end{gathered}
$$

where $\mathrm{J}$ is given by (16). Note that $\mathbf{C S}$ and $\mathbf{w}$ refer to the column vectors that contain the decision variables of the optimization problems (20)-(23). The upper and lower bounds of the weights are in the vectors $\overline{\mathbf{W}}$ and $\underline{\mathbf{w}}$. An elicitation technique for these bounds is discussed in Section V. In order to satisfy Eq. (23), the sum of the upper bounds must be greater than one and the sum of the lower bounds less than one, respectively. 
The vectors $\overline{\mathbf{C S}} i j^{\mathrm{t}}$ and $\mathbf{C S} i j{ }^{\mathrm{t}}$ in (21) contain the upper and lower bounds of the attributes that are defined by the feasible ranges of the state variables of the aircraft. The bounds are calculated by maximizing and minimizing (8)-(14) such that the values of the state variables are constrained according to the state and error vectors included in the information set of the transmitting team member. Because $\mathbf{T M} l$ is prioritizing $\mathbf{O P} j$ with respect to $\mathbf{T M} i$, the state vectors $\mathbf{O P} j^{\mathrm{t}}$ and $\mathbf{T M} i^{\mathrm{t}}$ as well as the error vectors $\mathbf{E O} l j^{\mathrm{t}}$ and $\mathbf{E T} l i^{\mathrm{t}}$ are used and the optimization problems to be solved can be written as

$$
\overline{\mathbf{C S}} i j^{\mathrm{t}} / \underline{\mathbf{C S}} i j^{\mathrm{t}}=\max _{\mathbf{T M}, \mathbf{O P}} / \min \mathbf{f}(\mathbf{T M}, \mathbf{O P})
$$

subject to

$$
\begin{aligned}
& \mathbf{O P} j^{\mathrm{t}}-\mathbf{E O} l j^{\mathrm{t}} \leq \mathbf{O P} \leq \mathbf{O P} j^{\mathrm{t}}+\mathbf{E O} l j^{\mathrm{t}}, \\
& \mathbf{T M} i-\mathbf{E T} l i^{\mathrm{t}} \leq \mathbf{T M} \leq \mathbf{T M} i+\mathbf{E T} l i^{\mathrm{t}},
\end{aligned}
$$

where $\mathbf{f}$ is given by (7) and $\mathbf{O P}$ and $\mathbf{T M}$ refer to the column vectors containing the decision variables. Due to the constraint (23) and the scaling of the single attribute value functions, the problems (20)-(23) and (24)-(26) provide interval importances that are limited to [0,1]. The time attribute in (20), that is the difference $\mathrm{t}-\mathrm{t} l j$, could also be treated by using an interval but it is not necessary since exact transmission and receiving times of earlier messages are known.

Interval importance indices for all the opponents perceived by the radar of the transmitting team member are determined by aggregating the interval importances. Following the techniques applied in additive value function models [23], the aggregation is carried out with the help of absolute and pairwise dominance. These dominance concepts do not produce a unique interval importance index in all possible combat states. Therefore, we introduce a new worst case aggregation rule.

Denote the interval importance index of $\mathbf{O P} j$ as $\left[\underline{\mathrm{Jj}}^{\mathrm{t}}, \overline{\mathrm{Jj}}^{\mathrm{t}}\right]$ and assume that it is composed of the interval importances $\left[\underline{\mathrm{J}}_{\underline{j}}^{\mathrm{t}}, \overline{\mathrm{J}} \boldsymbol{i}^{\mathrm{t}}\right], \forall i \in \mathrm{IK} l^{\mathrm{t}}$, determined by the transmitting aircraft $\mathbf{T M} l$. First, less essential importance intervals are eliminated with the absolute dominance rule. Importance interval $\left[\underline{\mathrm{J} k j}^{\mathrm{t}}, \overline{\mathrm{J} k j}^{\mathrm{t}}\right]$ is absolutely dominated by interval $\left[\underline{\mathrm{J} m j}^{\mathrm{t}}, \overline{\mathrm{J} m j}^{\mathrm{t}}\right]$, if $\underline{\mathrm{J} m j}^{\mathrm{t}} \geq \overline{\mathrm{J} k j}^{\mathrm{t}}$, see Fig. 7 .

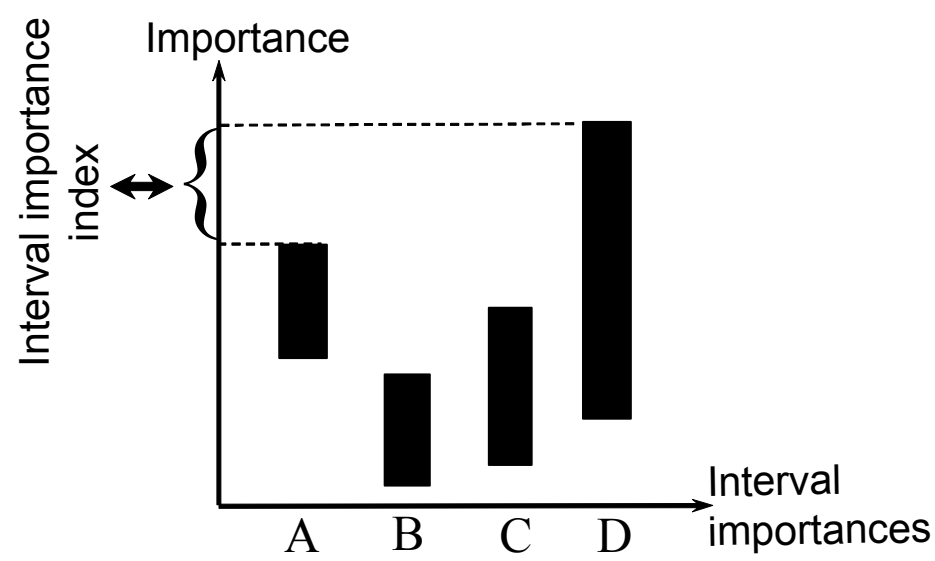

Fig. 7: B is absolutely dominated by A. C is pairwisely dominated by D. Applying the worst case aggregation rule, the upper bound of $\mathrm{D}$ and the upper bound of $\mathrm{A}$ form the interval importance index. 
The pairwise dominance rule is then applied for all remaining intervals $\left[\underline{\mathrm{J} m j}{ }^{\mathrm{t}}, \overline{\mathrm{J} m j}^{\mathrm{t}}\right]$ and $\left[\underline{\mathrm{J} k j}^{\mathrm{t}}, \overline{\mathrm{J} k j}^{\mathrm{t}}\right]$ which satisfy the inequalities $\overline{\mathrm{J} m j}^{\mathrm{t}}>\overline{\mathrm{J} k j}^{\mathrm{t}} \geq \underline{\mathrm{J} m j}^{\mathrm{t}}>\underline{\mathrm{J} k j}^{\mathrm{t}}$, see Fig. 7. According to pairwise dominance, the former interval dominates the latter one, if the importance $\mathrm{Jm} j^{\mathrm{t}}$ is greater than the importance $\mathrm{J} k{ }^{\mathrm{t}}$ for all the feasible values of the attributes and the weights. This is studied by solving the optimization problem

$$
\min _{\mathbf{C S} m, \mathbf{C S} k, \mathbf{w}}(\mathrm{J}(\mathbf{C S} m, \mathrm{t}, \mathrm{t} l j ; \mathbf{w})-\mathrm{J}(\mathbf{C S} k, \mathrm{t}, \mathrm{t} l j ; \mathbf{w}))
$$

subject to

$$
\begin{gathered}
\underline{\mathbf{C S} m j}^{\mathrm{t}} \leq \mathbf{C S} m \leq \overline{\mathbf{C S}} m j^{\mathrm{t}}, \\
\underline{\mathbf{C S} k j}{ }^{\mathrm{t}} \leq \mathbf{C S} k \leq \overline{\mathbf{C S} k j}^{\mathrm{t}}, \\
\underline{\mathbf{w}} \leq \mathbf{w} \leq \overline{\mathbf{w}}, \\
\|\mathbf{w}\|_{1}=1 .
\end{gathered}
$$

Here, the lower and upper bounds in (28) and (29) are determined by solving the problems (24)-(26) that contain the constraints defined by the appropriate state and error vectors. Pairwise dominance exits, if the optimal value of (27) is positive.

If two or more importance intervals still overlap each other, they are combined with the worst case aggregation rule. The upper bound of the importance index is then associated with the highest value of the upper bounds of the remaining importance intervals, i.e.,

$$
\overline{\mathrm{J}}^{\mathrm{t}}=\max _{i}\left\{\overline{\mathrm{J} i j}^{\mathrm{t}}\right\}
$$

and the lower bound with the lowest value of the upper bounds, i.e.,

$$
\underline{\mathrm{J}}^{\mathrm{t}}=\min _{i}\left\{\overline{\mathrm{J}} \boldsymbol{i j}^{\mathrm{t}}\right\} \text {. }
$$

An example of aggregation is shown in Fig. 7. By applying the aggregation process presented, the interval importance index for each opponent is obtained uniquely.

The final prioritization of the opponents is made by comparing the interval importance indices. A selection rule for this comparison is needed. Several rules have been adopted to analyze nondominated decision alternatives in additive value function models [23]. The following selection rules are suitable also for the signaling problem:

1. Maximax: Select the opponent $\mathbf{O P} k$ who has the greatest upper bound of the interval importance indices,

$$
\text { i.e., } \overline{\mathrm{J} k}^{\mathrm{t}} \geq \overline{\mathrm{J} j}^{\mathrm{t}}, \forall j \in \mathbf{I} l^{\mathrm{t}}, j \neq k
$$

2. Maximin: Select the opponent $\mathbf{O P} k$ who has the greatest lower bound of the interval importance indices,

$$
\text { i.e., } \underline{\mathrm{J} k^{\mathrm{t}}} \geq \underline{\mathrm{J} j}{ }^{\mathrm{t}}, \forall j \in \mathbf{I} l^{\mathrm{t}}, j \neq k
$$

3. Central values: Select the opponent $\mathbf{O P} k$ who has the greatest center of the interval importance indices,

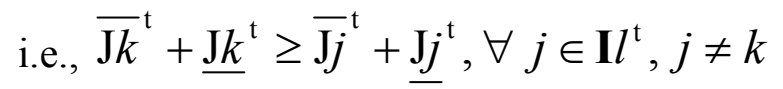

The first criterion describes the behavior of a risk averse decision maker, because the prioritization is based on the worst possible state of the combat. The maximin criterion utilizes the best possible state and thus it represents a risk prone decision maker. A risk neutral decision maker can be assumed to behave according to the central values criterion. An example on the use of these criteria is shown in Fig. 8. 


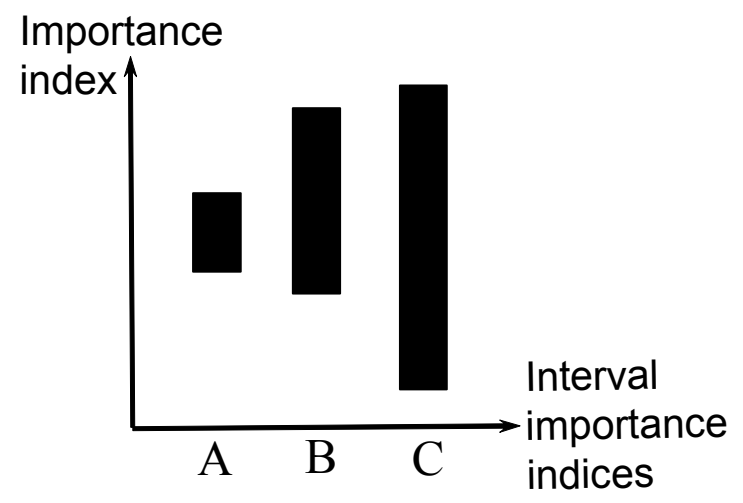

Fig. 8: The maximax rule leads to the selection of $\mathrm{C}$, the maximin $\mathrm{A}$, and the central values $\mathrm{B}$, respectively.

The moving average over the importance indices associated with earlier transmitted messages is still a viable importance index for the transmitting team member, if the same selection rule in the prioritization of the opponents is applied at all transmission instants. The index of $\mathbf{T M} l$ at time $\mathrm{l}_{\mathrm{k}}$ is given by

$$
\mathrm{JT}^{1_{\mathrm{k}}}=\frac{1}{\mathrm{~L}} \mathrm{v}_{\mathrm{t}}^{\mathrm{tm}}\left(1_{\mathrm{k}}-\mathrm{t} l\right) \sum_{\mathrm{m}=1}^{\mathrm{L}} \mathrm{J}^{\mathrm{l}_{\mathrm{k}-\mathrm{m}}},
$$

where the index of the message transmitted earlier is denoted by $\mathrm{J}^{\mathrm{l}_{\mathrm{k}-\mathrm{m}}}$. It is equal to $\mathrm{JT} l^{1_{\mathrm{k}-\mathrm{m}}}$ corresponding to the message on own state information, or equal to $\max \left\{\overline{\mathrm{J} j}^{1_{\mathrm{k}-\mathrm{m}}}\right\}, \max \left\{\underline{\mathrm{J} j}^{1_{\mathrm{k}-\mathrm{m}}}\right\}$ or $\max \left\{\overline{\mathrm{J}}^{1_{\mathrm{k}-\mathrm{m}}}+\underline{\mathrm{J}}^{1_{\mathrm{k}-\mathrm{m}}}\right\}, \forall j \in \mathbf{I} l^{1_{\mathrm{k}-\mathrm{m}}}$, when the maximax, maximin or central values selection rule is used.

\section{B. Solution of the Signaling Problem}

At each transmission instant, the state information included in the message to be relayed is now found by comparing the importance index of the transmitting team member and the most important opponent that is identified with the interval importance indices and an appropriate selection rule. To summarize, the team optimal signaling strategy is solved by the following procedure:

1. Set $\mathrm{l}=1$ and $\mathrm{k}=1$.

2. At $\mathrm{t}=(1-1)+(\mathrm{k}-1) \mathrm{M}$, determine the interval importance $\left[\underline{\mathrm{J}}^{\mathrm{t}}{ }^{\mathrm{t}}, \overline{\mathrm{J}} \boldsymbol{j}^{\mathrm{t}}\right] \forall i \in \mathbf{I K} l^{\mathrm{t}}, \forall j \in \mathbf{I} l^{\mathrm{t}}$ by solving (20)-(23) with $\mathbf{I O} l^{\mathrm{t}}$.

3. Determine the interval importance index $\left[\underline{\mathrm{J} j}^{\mathrm{t}}, \overline{\mathrm{J} j}^{\mathrm{t}}\right]$ for all $j \in \mathbf{I} l^{\mathrm{t}}$ by aggregating the interval importances with the aggregation process.

4. Select the most important opponent by ranking the interval importance indices with the given selection rule.

5. Determine the point-estimate importance index for the most important opponent by choosing the upper bound, the lower bound or the central value of its interval importance index according to the given selection rule.

6. Select the most important aircraft by comparing the point-estimate interval importance index of the most important opponent and the importance index of the transmitting team member TM $l$ given by (34).

7. Transmit the data link message containing the state information of the most important aircraft.

8. If $1<\mathrm{M}$, set $\mathrm{l}=1+1$ and return to 2 . Otherwise, go to 9 .

9. If $\mathrm{k}=\mathrm{K}$, terminate. Otherwise, set $\mathrm{l}=1, \mathrm{k}=\mathrm{k}+1$ and return to 2 . 
The interval analysis and the above solution procedure can also be used in cases in which either only the attributes or the weights are expressed as intervals. If all the attributes and the weights are quantified by point-estimates, interval importances and interval importance indices reduce to the measures presented in Section III.

\section{IPAC SOFTWARE}

The prioritization approach presented in Sections III and IV is implemented in the Information Prioritization in Air Combat (IPAC) software. It is a Matlab-based [18] tool that simulates and visualizes trajectories and signaling of the players during an M-on-N air combat game. IPAC consists of a graphical user interface and Matlab's M-files in which all the computation is performed. The optimization problems imposing interval importance indices on the opponents are solved with the CONSTR routine of the Matlab's Optimization Toolbox [9] that utilizes the sequential quadratic programming method [5]. IPAC also provides aids for extracting single attribute value functions and their weights from the experts.

Before beginning a simulation run, the user of IPAC gives the general simulation settings such as the numbers of the team members and the opponents, the duration of the combat and the time interval between subsequent transmissions. In addition, the maximum viewing angle and reach of the team members' radar are fixed. Then, the user must specify the general prioritization settings. The priorities of the aircraft can be calculated using point-estimate or interval importance indices. When the intervals are used, one can choose an appropriate selection rule as well as the rate of change for error terms. For comparison purposes, the aircraft can also be ranked according to a commonly used prioritization measure that is described in Section VI.

Once the simulation and prioritization settings are given, the user defines single attribute value functions and their weights. In IPAC, the shape of each single attribute function is specified graphically with a mouse. The shape can be arbitrary and hence the user can apply different elicitation techniques. An alternative is to construct a single attribute value function simply by assessing the form of the function. In the bisection method [15], attribute levels are compared pairwise. The user chooses a midpoint between two levels such that the improvement of the value from the one attribute level to the midpoint is equal to the improvement of the value from the midpoint to the another attribute level. This gives two attribute intervals whose midpoints are selected in a similar way. The procedure is repeated until the single attribute value function is defined with desired accuracy. In the difference standard sequence technique [15], the user determines a set of attribute levels such that the increment of the value is equal between all the successive attribute levels.

In IPAC, point-estimates of weights are elicited by following a procedure in which the user gives ten points to the least important term of the value function. The rest of the terms are assigned points higher than ten reflecting their relative importance. The resulting weights are normalized automatically in order to sum to one. The procedure of this type is an extension of the simple multiattribute rating technique (SMART) [7], [8] that is used widely for assessing weights of additive value models. In the assessment of interval weights, the user first selects a reference term that is assigned with a point-estimate weight, e.g., ten points. Then, the other terms are compared to the reference term and weight intervals are specified. When all the weight intervals are determined, IPAC normalizes the interval weights such that the sum of their upper bounds is greater than one and the sum of the lower bounds less than one, respectively. The elicitation procedure described is similar to the interval SMART weighting method [20] applied in decision models based on an additive value function.

In the simulation run of IPAC, the aircraft follow a guidance law in which a team member is ordered to pursue a particular opponent and vice versa. The user must only define the initial states of the aircraft, see 
Fig. 9 (a). The aircraft can also fly along given trajectories saved earlier in a file or along a straight line with a constant velocity and direction of flight. If a team member does not get a radar observation or a data link message about a particular aircraft, IPAC extrapolates the position of this aircraft based on the latest received information on its velocity and direction of flight. The positions of the team members and the opponents are visualized on a map display by moving symbols. Their velocity vectors imply direction of flight and speed, see Fig. 9 (b). The colors of the symbols indicate the transmitting team member and the aircraft whose information is being relayed. The graphical representation of the combat state is easily understood because the map and the symbols in the IPAC's user interface are similar to those used on the display of a radar. The order of importance of the aircraft calculated by each team member is also shown on the screen, see Fig. 9 (b).

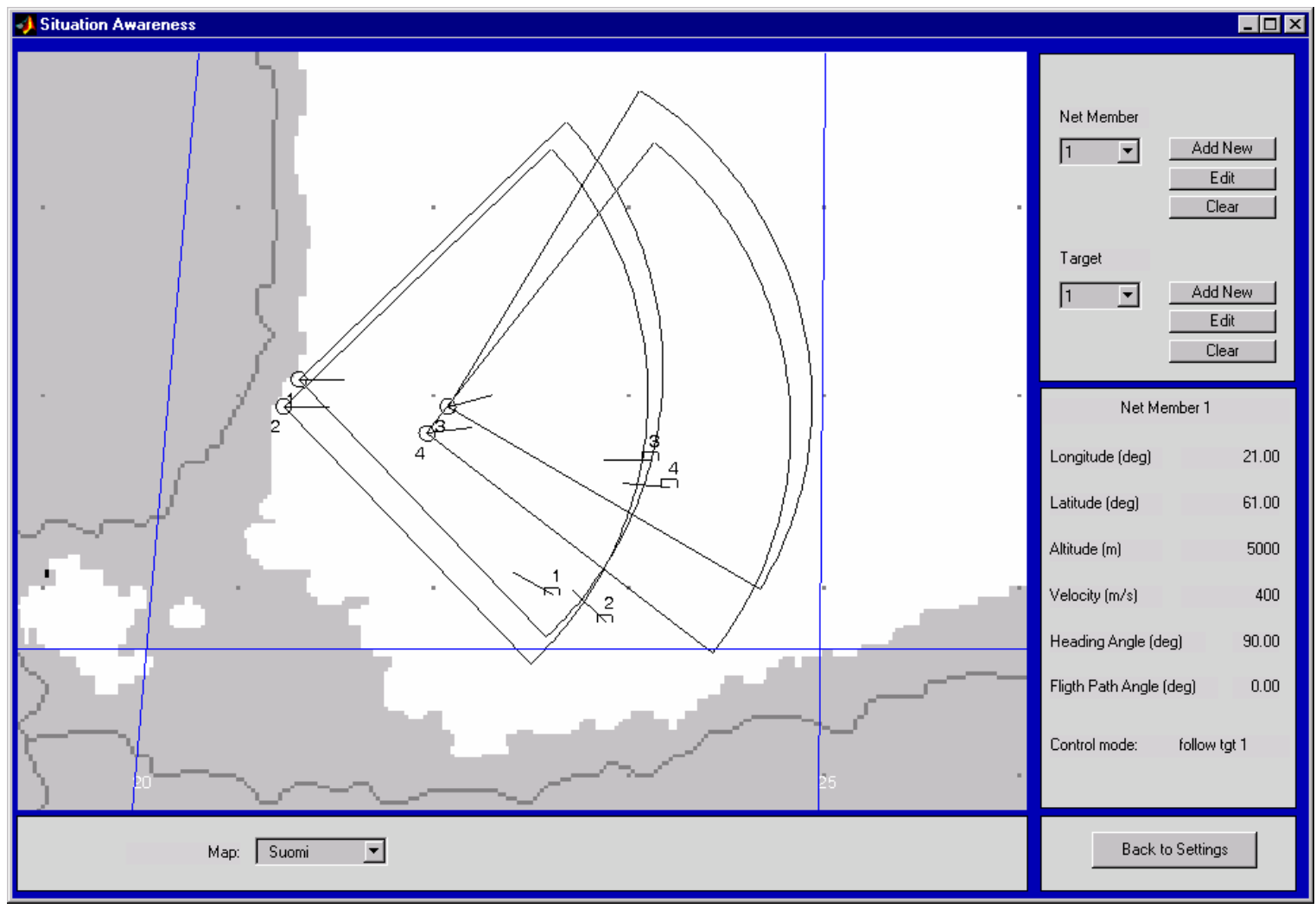

Fig. 9 (a): Specification of the initial states of the aircraft.

During the simulation of an air combat game, all the results consisting of the states of the aircraft, importances and importance indices are recorded and saved into a file. The user can study simulation results by visualizing them or viewing the numerical values. For example, importance indices are plotted as a function of time. Furthermore, IPAC offers several measures for the quality of signaling strategies. For instance, one can calculate the cumulative time a team member is not aware of opponents that are in advantageous positions for using their weapons. It is also possible to load the results of earlier simulations and to rerun old air combat games. Then, the user can compare signaling strategies that are obtained in the same air combat scenario with the different parameters of the value function or with different prioritization approaches. 


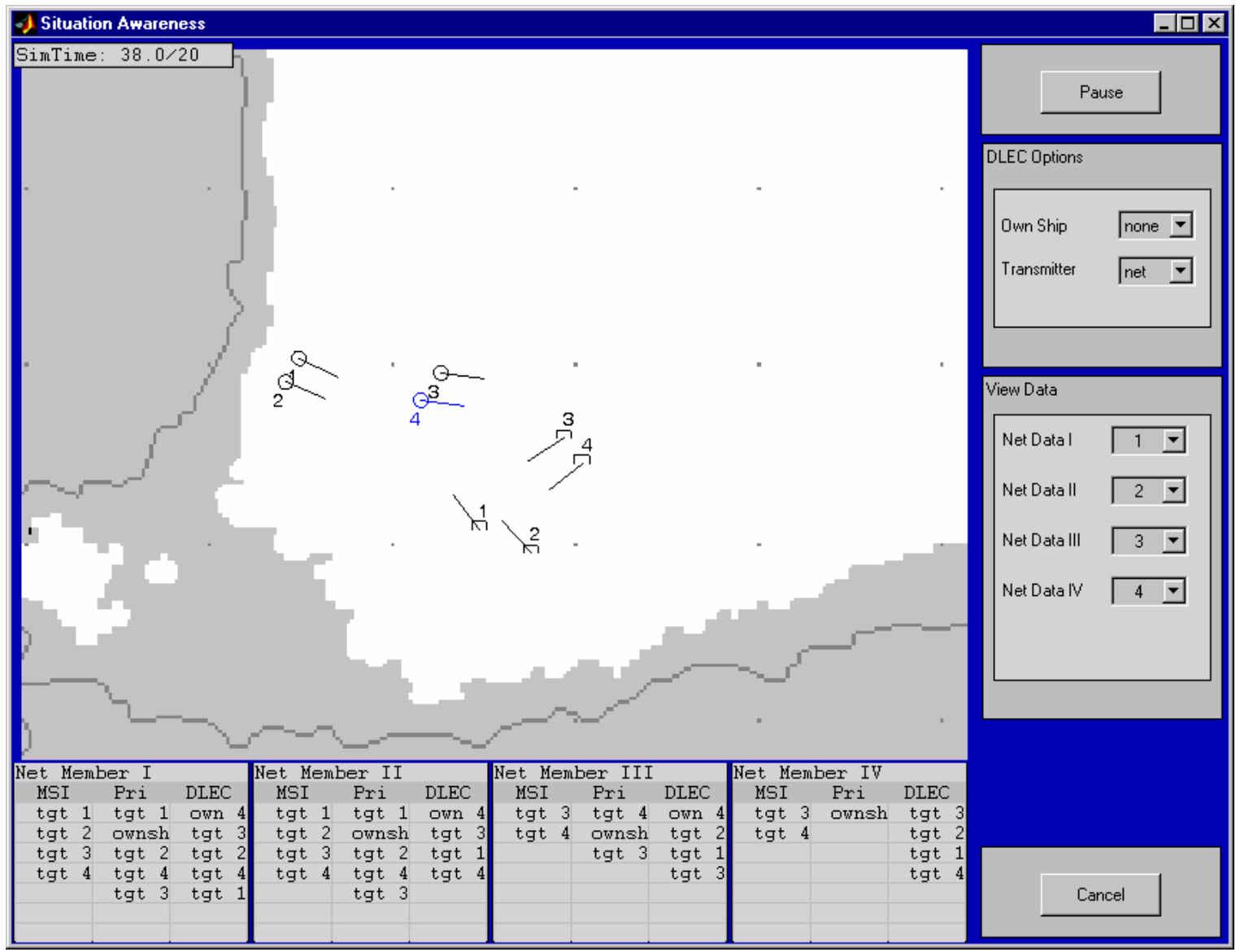

Fig. 9 (b): Visualization of the air combat game.

\section{SIMULATION EXAMPLES}

In this section, the presented prioritization approach is illustrated by simulating the trajectories of aircraft and the transmissions of data link messages in a three-on-three air combat game. The usability of the approach is demonstrated and compared to two existing prioritization approaches. In this comparison, perfect state information of the aircraft as well as point-estimate weights of the value function are used. In addition, an example sensitivity analysis with respect to the weights of the value function is conducted by applying interval analysis. The simulation examples are computed with the above described IPAC software.

\section{A. Air Combat Game}

The players of the air combat game are grouped into the friendly team consisting of the team members TM1, TM2, and TM3, and the adversary team consisting of the opponents OP1, OP2, and OP3. The trajectories of the players are shown in Fig. 10. All the players fly at a constant altitude of $5000 \mathrm{~m}$. The constant velocity of the team members as well as of OP2 and OP3 is $350 \mathrm{~m} / \mathrm{s}$, and the constant velocity of $\mathrm{OP} 1$ is $300 \mathrm{~m} / \mathrm{s}$. The total duration of the game is $99 \mathrm{~s}$ and the time interval between the transmissions of two successive messages $3 \mathrm{~s}$, respectively. The maximum memory time of the team members is set to $t_{\max }=$ $45 \mathrm{~s}$. The maximum reach of the team members' radar is $200 \mathrm{~km}$ and the maximum viewing angle measured from the velocity vector is $75^{\circ}$. 


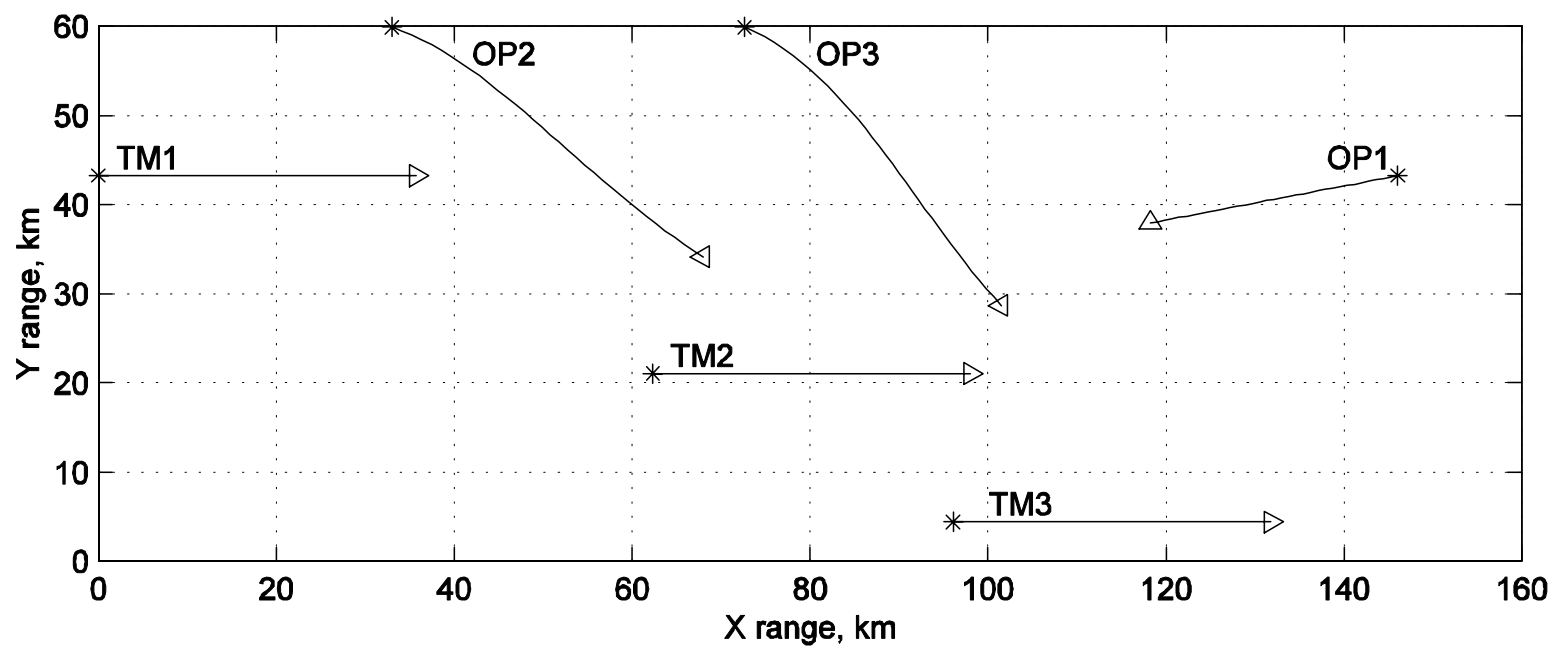

Fig. 10: The trajectories of the players in the example air combat game. * refers to the initial states and $D$ to the terminal states, respectively.

The trajectories are chosen such that one can qualitatively conclude the importance order of the opponents that is based on information acquired from the radars of the team members. In the example, TM1 receives radar observations on all the opponents throughout the game. TM2 is aware of OP1 and OP3 but its radar cannot track OP2. OP1 is in the radar beam of TM3 until $85 \mathrm{~s}$, and thereafter TM3 gets information about the opponents from data link messages only. OP2 can be considered as the most important opponent because it ends up flying behind TM2 and TM3. OP3 is the second important opponent since it is not tracked by the radar of TM3. The least important opponent is OP1. In the beginning of the game, all the friendly team members know each others' states. After the combat has proceeded 45 s, i.e., the maximum memory time of the team members, messages about the states of the team members are required.

\section{B. Parameters of the Value Function}

In the simulation examples, illustrative single attribute value functions and their weights are used. The shapes of the single attribute value functions sketched by the authors are shown in Fig. 11. The increasing value function for the deviation angle is chosen because the accuracy of a radar reduces as the deviation angle increases. A high importance component is associated with the opponent that cannot be seen by the radar of the team member. The value function of the angle off is also increasing because large angles refer to situations in which the opponent can see the team member with the radar. The importance component resulting from the distance between two players is assumed to increase when the distance decreases. The increasing value function of the approaching velocity produces high importance if the players are approaching each other and low importance in an inverse situation. The maneuvering of the opponent should enhance its importance. Therefore, the value function of the acceleration is constructed such that it gives a high importance component to the opponent flying with a high acceleration. The value function of the time attribute increases with time. 

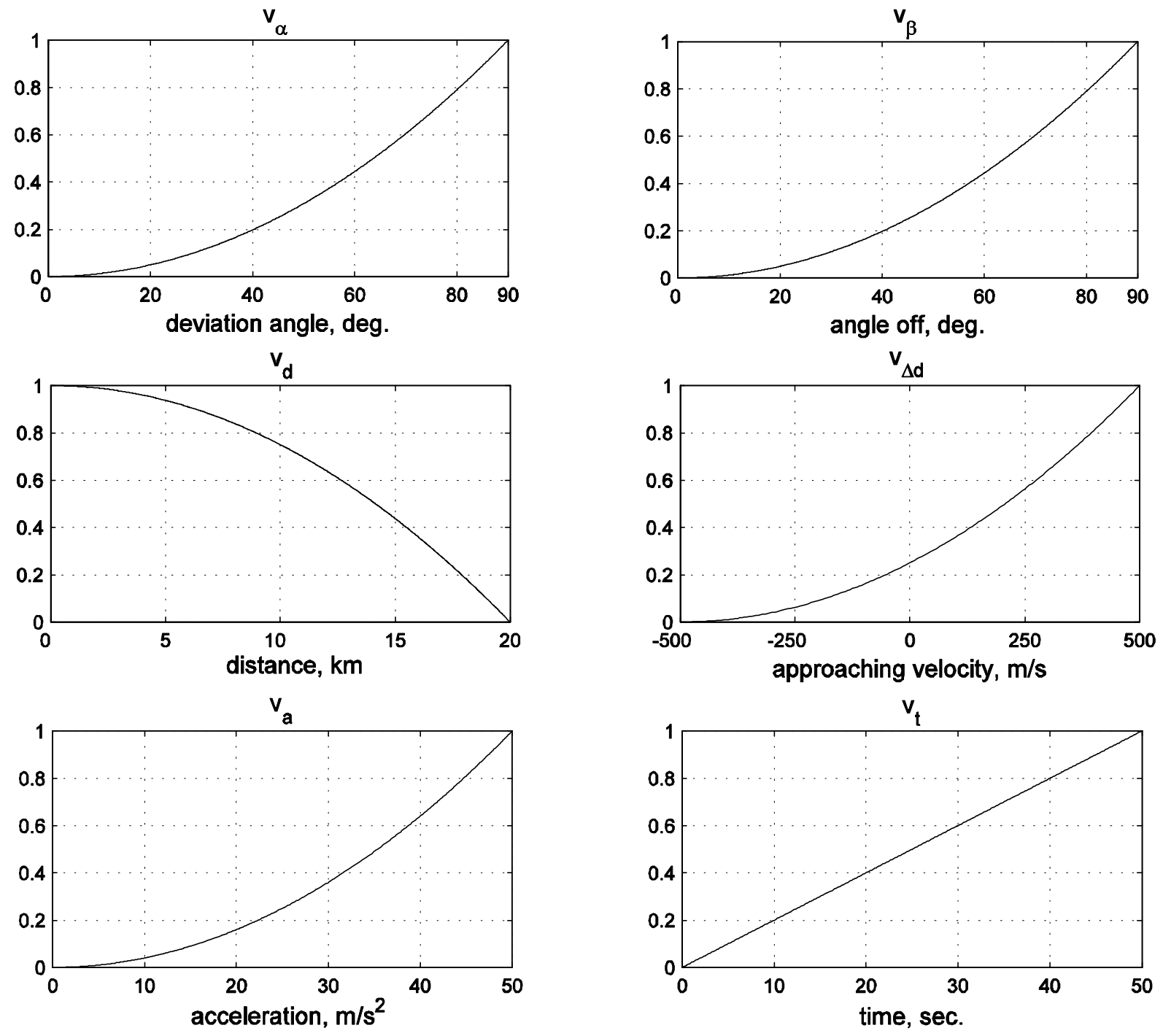

Fig. 11: The single attribute value functions.

The altitude and velocity differences are ignored in the simulation examples because all the players fly at the same altitude and only the velocity of OP1 differs from the velocity of the other players. In general, the value functions of the altitude and velocity differences should be increasing since high levels of these attributes refer to the energy advantage of the opponent which must increase importance.

The point-estimate weights of the overall value function assessed by the authors are presented in Table 1 . The highest weights are set to the terms including three attributes. These terms produce high importance when the opponent is reaching or has reached the team member's tail. In such a combat situation, the team member cannot see the opponent with the radar and, on the other hand, the opponent is in an advantageous position for using its weapons. The second highest weights are associated with the two attribute terms containing the deviation angle. They give high importance to the opponent that is beyond the range of the team member's radar regardless of the opponent's direction of flight. The two attribute term related to the acceleration is assumed to be less important than the other ones. The next important terms take into account the angle off. They contribute to importance when the opponent observes the team member with the radar. The single attribute terms are assigned with the lowest weights. The major part of the importance results from these terms when the deviation angle and the angle off are close to $0^{\circ}$. Then, the team member is flying behind the opponent and their flight directions are similar. In the combat situation of this type, the opponent is not important and thus the low weights are justified. 


\begin{tabular}{|l|c|c|c|c|c|c|c|}
\hline Single attribute terms & $\alpha$ & $\beta$ & $\mathrm{d}$ & $\Delta \mathrm{d}$ & $\Delta \mathrm{v}$ & $\Delta \mathrm{h}$ & $\mathrm{a}$ \\
\hline Weight & 0.0564 & 0.0282 & 0.0188 & 0.0188 & 0 & 0 & 0.013 \\
\hline Multiattribute terms & $\alpha \mathrm{d}$ & $\alpha \Delta \mathrm{d}$ & $\alpha \mathrm{a}$ & $\beta \mathrm{d}$ & $\beta \Delta \mathrm{d}$ & $\alpha \beta \mathrm{d}$ & $\alpha \beta \Delta \mathrm{d}$ \\
\hline Weight & 0.141 & 0.1504 & 0.0564 & 0.0846 & 0.0752 & 0.188 & 0.1692 \\
\hline
\end{tabular}

Table 1: The weights of the value function.

\section{Alternative Prioritization Approaches}

In the simulation examples, the value function based prioritization approach, hereafter referred to as the value function approach, applies the worst case aggregation, i.e., Eq. (18), for composing importances into importance indices. It is compared with two approaches that utilize a measure used in the data link system for determining one-on-one importance between a team member and an opponent. The measure is calculated by dividing the approaching velocity of the players by their distance. It gives low importance for opponents that are far away and receding from the team member. High importance is associated with an approaching opponent that is close to the team member.

In the rate of range approach, the opponents are prioritized only with respect to the transmitting team member. The states of the other friendly team members are not taken into account in the prioritization. At each transmission instant, the above mentioned measure is calculated for each opponent that is tracked by the radar of the transmitting team member. The resulting measures are used as importance indices that determine information included in data link messages. The transmitting member sends its own state information only if it is not able to track any opponents.

The team rate of range approach utilizes the same measure as above for determining one-on-one importances, but the transmitting team member evaluates the importance for each combination of tracked opponents and known team members. The highest one-on-one importance of each opponent is used as the importance index, and the opponents are ranked based on these indices. The importance index for the transmitting member is obtained by calculating the moving average of the importance indices associated with the earlier relayed messages.

\section{Comparison of the Prioritization Approaches}

First, the example air combat game is simulated by prioritizing the players with the rate of range approach. The importance indices determined by each team member are shown as a function of time in Figs. 12 (a)(c). The players whose state information is included in the data link messages at each transmission instant are presented in Fig. 12 (d). The importance indices calculated by TM1 imply that OP1 is the most important opponent throughout the game. A reason for this is that the approaching velocity of OP1 towards TM1 is largest. TM2 prefers OP1 to OP3 until $21 \mathrm{~s}$ and after that OP3 becomes the most important opponent. TM3 calculates the importance index only for OP3 because the other opponents are not tracked by the radar.

The majority of the transmitted messages concern OP1 (see Fig. 12 (d)) although it is the least important opponent. There are also messages including information about OP3. However, these messages improve the situation awareness of TM3 only. The biggest drawback of the transmission sequence of the opponents' state information is that the team members do not relay information about OP2 that is qualitatively the most important opponent. TM3 is the only team member who transmits its own state information. These messages are sent after $85 \mathrm{~s}$ when TM3 ceases the tracking of the opponents. Due to the lack of the transmissions of TM1's and TM2's state information, TM3 becomes unaware of TM1 and TM2 as well as TM2 becomes unaware of TM1 after the maximum memory time $45 \mathrm{~s}$. Based on this as well as on the need 
for messages on OP2, one can conclude that the use of the rate of range approach leads to the inadequate situation awareness of the team members.
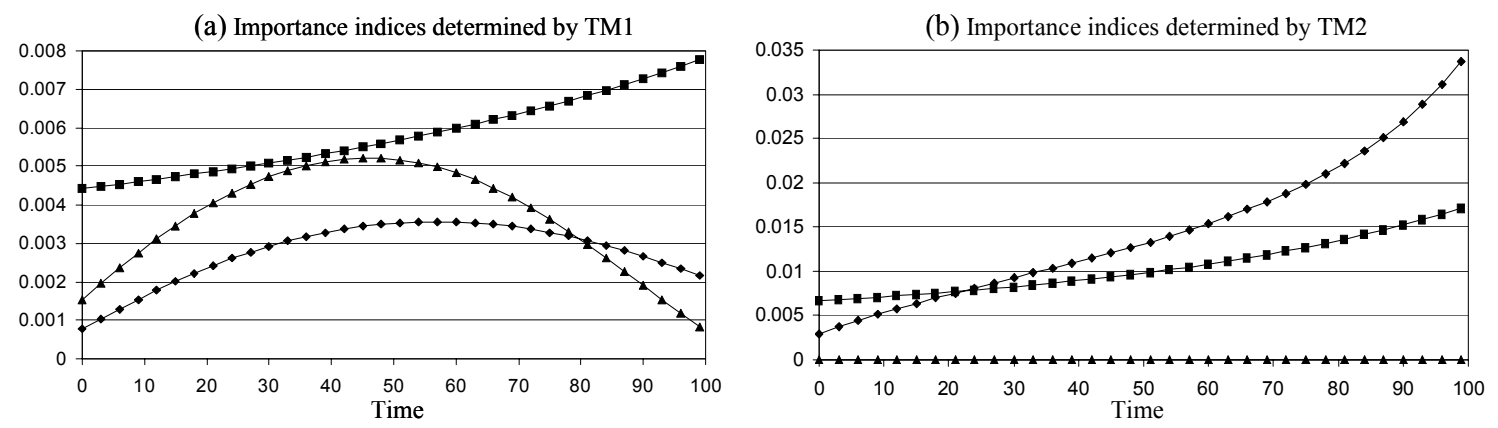

(c) Importance indices determined by TM3
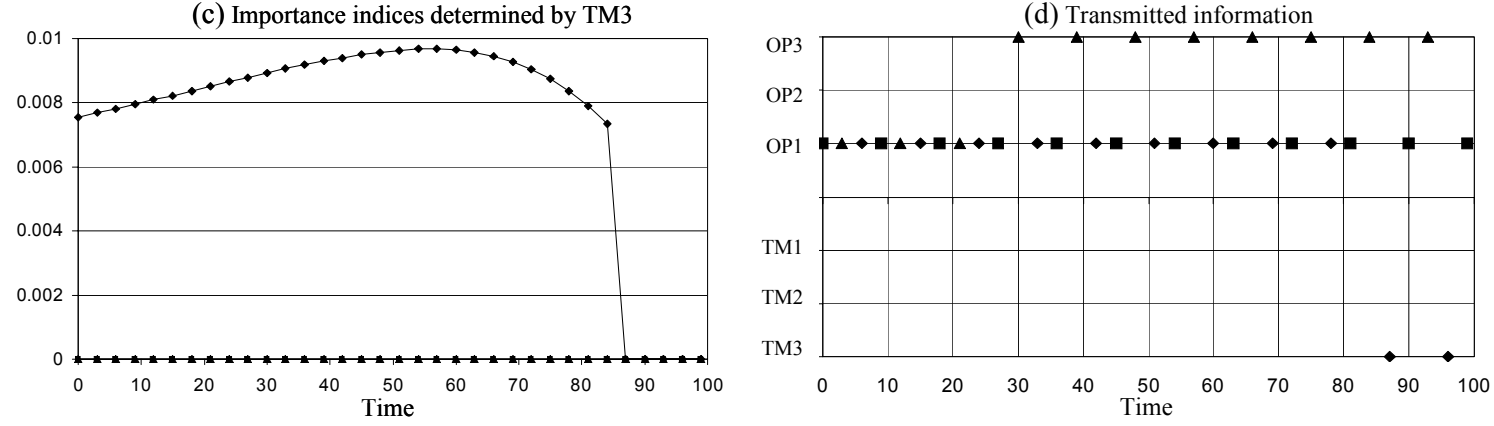

Fig. 12: Importance indices determined by (a) TM1, (b) TM2, and (c) TM3 with the rate of range approach. $\boldsymbol{\square}$ refers to OP1, $\boldsymbol{\Delta}$ to OP2, and $\boldsymbol{t}$ to OP3, respectively. (d) Transmitted state information at each transmission instant. corresponds to the transmission of TM1, $\mathbf{\Delta}$ to TM2, and to TM3, respectively.

Next, the prioritization is conducted with the team rate of range approach. The resulting importance indices and the sequence of the transmitted information are shown in Figs. 13 (a)-(d). According to the importance indices determined by TM1, OP1 is the most important opponent until $27 \mathrm{~s}$. After that, the highest priority is associated with OP3. The importance index of OP2 is again lowest. The importance index of TM1 is low during the combat and hence its state information is not relayed. TM2 gives the highest importance index to OP1 until $66 \mathrm{~s}$ though OP3 would be more important because it is not tracked by TM3. After $66 \mathrm{~s}$, the importance index of TM2 dominates and thus it transmits own state information. TM3 prioritizes OP1 with respect to TM1 and TM2 as far as OP1 is within the range of the TM3's radar. Because TM1 and TM2 do not transmit their own state information before $45 \mathrm{~s}$, they drop from the memory of TM3 at this time point, and TM3 begins to transmit its own state information. At $t=75 \mathrm{~s}, \mathrm{TM} 2$ sends a message about its own state and TM3 becomes aware of it. The messages about OP1 are sent until $85 \mathrm{~s}$ when OP1 flies beyond the range of the TM3's radar.

The importance indices of the transmitting team members are calculated based on the moving average. They increase in time (see Figs. 13 (a)-(c)) but the index of TM2 is the only one that starts to dominate. Hence, TM2 sends its own state information after $66 \mathrm{~s}$ but such messages would have been needed earlier because TM3 is unaware of TM2 from $45 \mathrm{~s}$ to $75 \mathrm{~s}$. TM1 does not transmit its own state information at all and hence it is not known by TM2 and TM3 after 45 s. 
(a) Importance indices determined by TM1

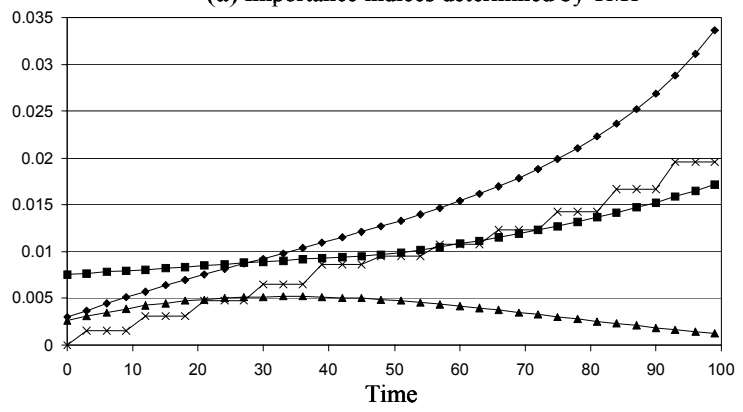

(c) Importance indices determined by TM3

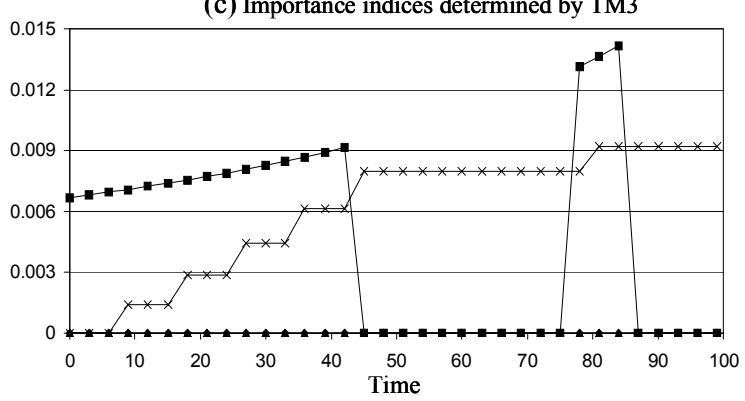

(b) Importance indices determined by TM2

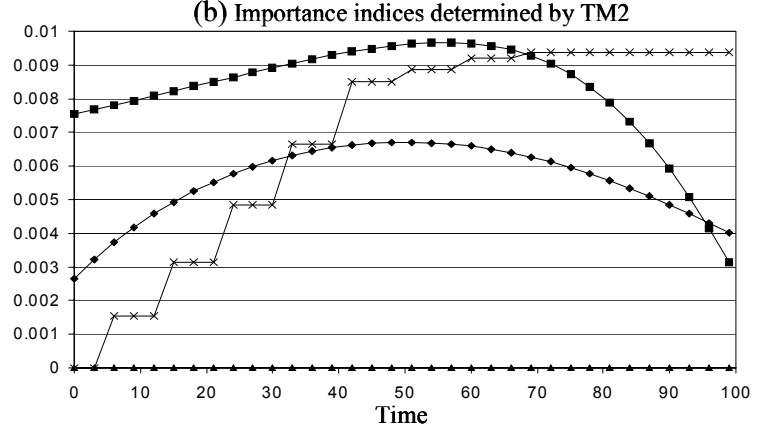

(d) Transmitted information

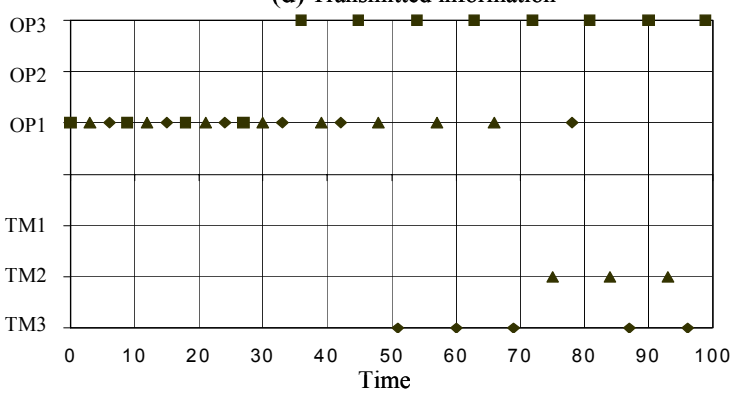

Fig. 13: Importance indices determined by (a) TM1, (b) TM2, and (c) TM3 with the team rate of range approach. refers to OP1, $\boldsymbol{\Delta}$ to OP2, to OP3, and X to the transmitting team member, respectively. (d) Transmitted state information at each transmission instant. $\mathbf{a}$ corresponds to the transmission of TM1, $\boldsymbol{\Delta}$ to $\mathrm{TM} 2$, and $\diamond$ to TM3, respectively.

Although the opponents are now prioritized with respect to the whole friendly team, the resulting signaling strategy is similar to the strategy obtained with the rate of range approach, see Figs. 12 (d) and 13 (d). The only improvements are three messages about TM2 in the end of the simulation. The biggest shortcoming is still the lack of the transmitted information on OP2. Most of the messages are related to OP1 though these are useless because all the team members track OP1 for almost the whole game. In addition, the first message about OP 3 is relayed at $\mathrm{t}=36 \mathrm{~s}$ and hence TM 3 is unaware of it until then. To summarize, the signaling strategy produced by the team rate of range approach gives a slightly better situation awareness to the friendly team than the strategy determined by the rate of range approach. However, TM2's and TM3's knowledge of the combat state is still incomplete.

In the next simulation run, the players are prioritized using the value function approach. The evolution of the importance indices in time and the transmitted information at each transmission instant are shown in Figs. 14 (a)-(d). The importance indices determined by TM1 show that OP2 and OP3 are now prioritized as the most important opponents. Most of the messages transmitted by TM1 are related to OP2. Otherwise the other team members would not be aware of OP2 and, on the other hand, TM2 concentrates on the transmissions about OP3. OP1 and OP3 have the highest importance indices calculated by TM2. TM2 sends more frequently messages about OP3 than messages about OP1 because the majority of the messages sent by TM3 are related to OP1. In addition to the messages about OP1, TM3 transmits information about its own state. Because of the messages of TM3 about OP1, the other team members do not have to send information of this type except in the end of the combat when OP1 flies beyond the range of the TM3's radar. 
(a) Importance indices determined by TM1

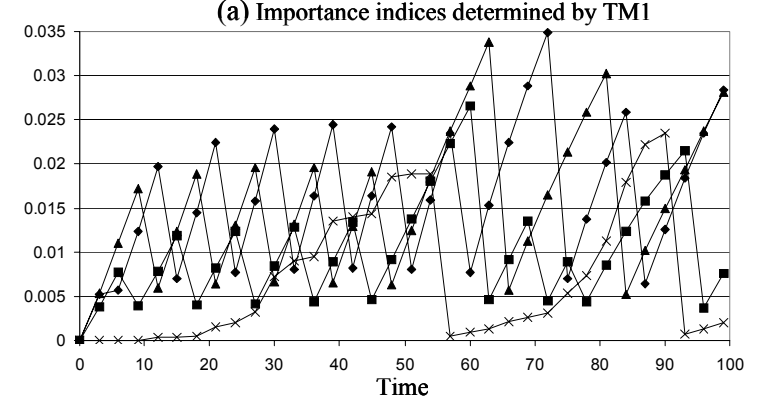

(c) Importance indices determined by TM3

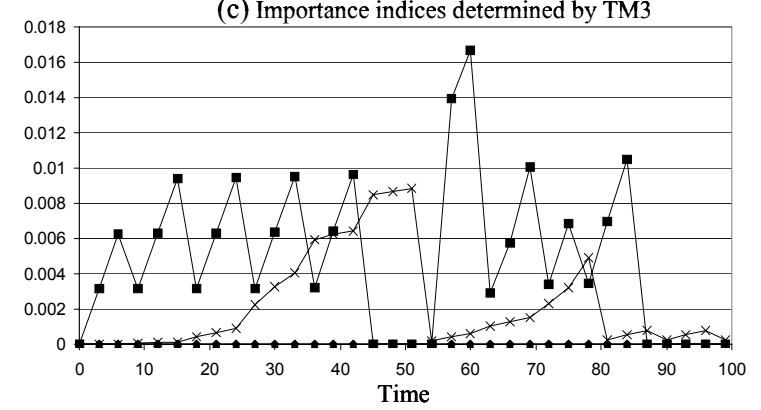

(b) Importance indices determined by TM2

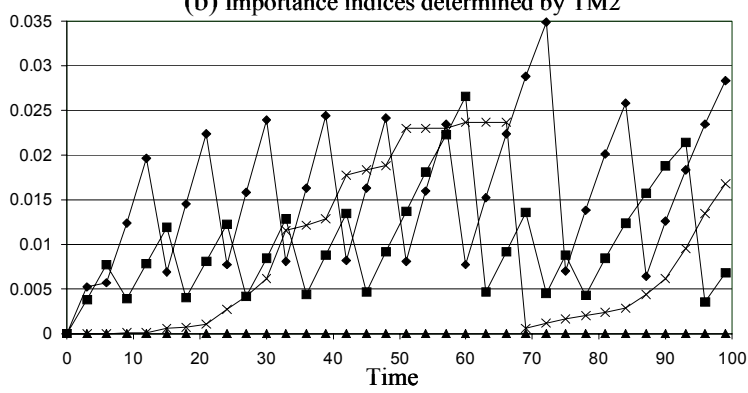

(d) Transmitted information

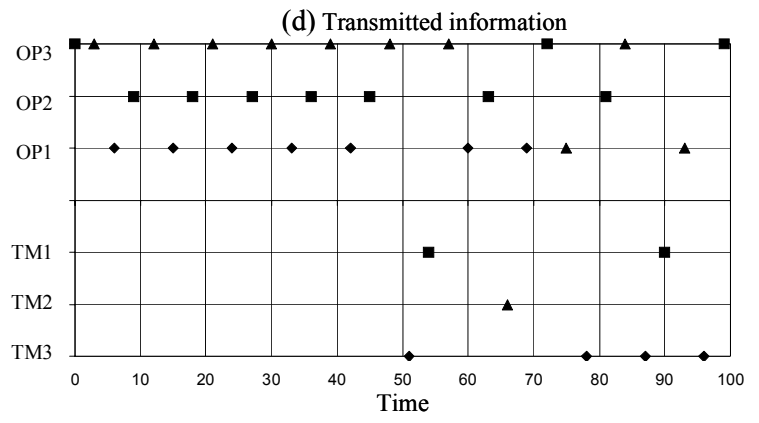

Fig. 14: Importance indices determined by (a) TM1, (b) TM2, and (c) TM3 with the value function

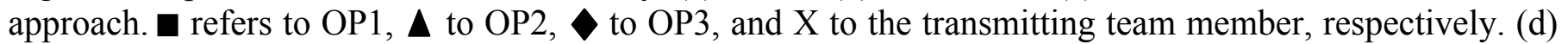
Transmitted state information at each transmission instant. corresponds to the transmission of TM1, $\boldsymbol{\Delta}$ to $\mathrm{TM} 2$, and $\checkmark$ to TM3, respectively.

The impact of the time attribute of the value function (16) on the importance indices of the opponents can be seen in Figs. 14 (a)-(c). After the transmission or receiving of the message about a particular opponent, the importance index of this opponent decreases rapidly and then begins to increase again. In this way, the prioritization approach allows transmissions about opponents that are less important with respect to the combat state but whose state information has not been transmitted for some time. The phenomenon of this type does not occur if the alternative prioritization approaches are used. The plots of the importance indices are smooth and do not fluctuate at transmission instants, see Figs. 12 (a)-(c) and 13 (a)-(c).

Figs. 14 (a)-(c) show the contribution of the time attribute to the moving average importance index of the transmitting team members. The indices decrease rapidly at each transmission instant of own state information. The moving average index is now appropriate because all the team members transmit their own state information after $45 \mathrm{~s}$. However, TM2 and TM3 are unaware of the TM1's state from $45 \mathrm{~s}$ to $54 \mathrm{~s}$ and TM3 is unaware of the TM2's state from $45 \mathrm{~s}$ to $66 \mathrm{~s}$. This indicates that the growth rate of the time's single attribute value function in Eq. (19) should be higher.

The value function approach gives the signaling strategy in which information about all the opponents is included in the transmitted messages. The messages about OP2 are also transmitted. They are missing in the strategies determined by the alternative approaches. In addition, each friendly team member transmits its own state information. Hence, all the team members are aware of each other after the game has proceeded $66 \mathrm{~s}$. Due to the messages about all the opponents as well as about all the team members, the value function approach produces a good situation awareness for the whole friendly team.

\section{E. Sensitivity Analysis}

As an example sensitivity analysis, the impacts of the weights of the value function on signaling strategies are studied. The team optimal signaling strategy obtained in the previous subsection with the value function approach is used as a reference strategy, and the point-estimate weights presented in Table 1 as reference weights, respectively. The sensitivity analysis is carried out such that the air combat game is simulated 
several times. In each simulation run, one weight of the value function is allowed to vary between a given range and all other weights are kept at their reference point-estimates. The range of each interval weight is equal. During the simulations, the players are prioritized with the interval approach introduced in Section IV.

If the variation of a particular weight leads to a different signaling strategy than the reference strategy, one can conclude that this weight is crucial. The comparison of the quality of signaling strategies could be made, e.g., by using measures available in the IPAC software. However, in this example, the comparison is based on the change of transmitted information. It is measured by calculating the percentual value of messages whose contents differ from the reference strategy.

The results of the sensitivity analysis are shown in Fig. 15. Although the weight of the term d, a, $\alpha \mathrm{d}, \alpha \mathrm{a}$, $\beta \mathrm{d}, \alpha \beta \mathrm{d}$, or $\alpha \beta \Delta \mathrm{d}$ is varied, the resulting signaling strategy is the same as the reference strategy. When the weight of the term $\alpha$ is altered, the change of the transmitted information is $3 \%$. In further analysis, the weights of these terms of the value function can be left at their reference point-estimates because the signaling strategy is insensitive with respect to them. In this way, less important weights are identified and singled out.

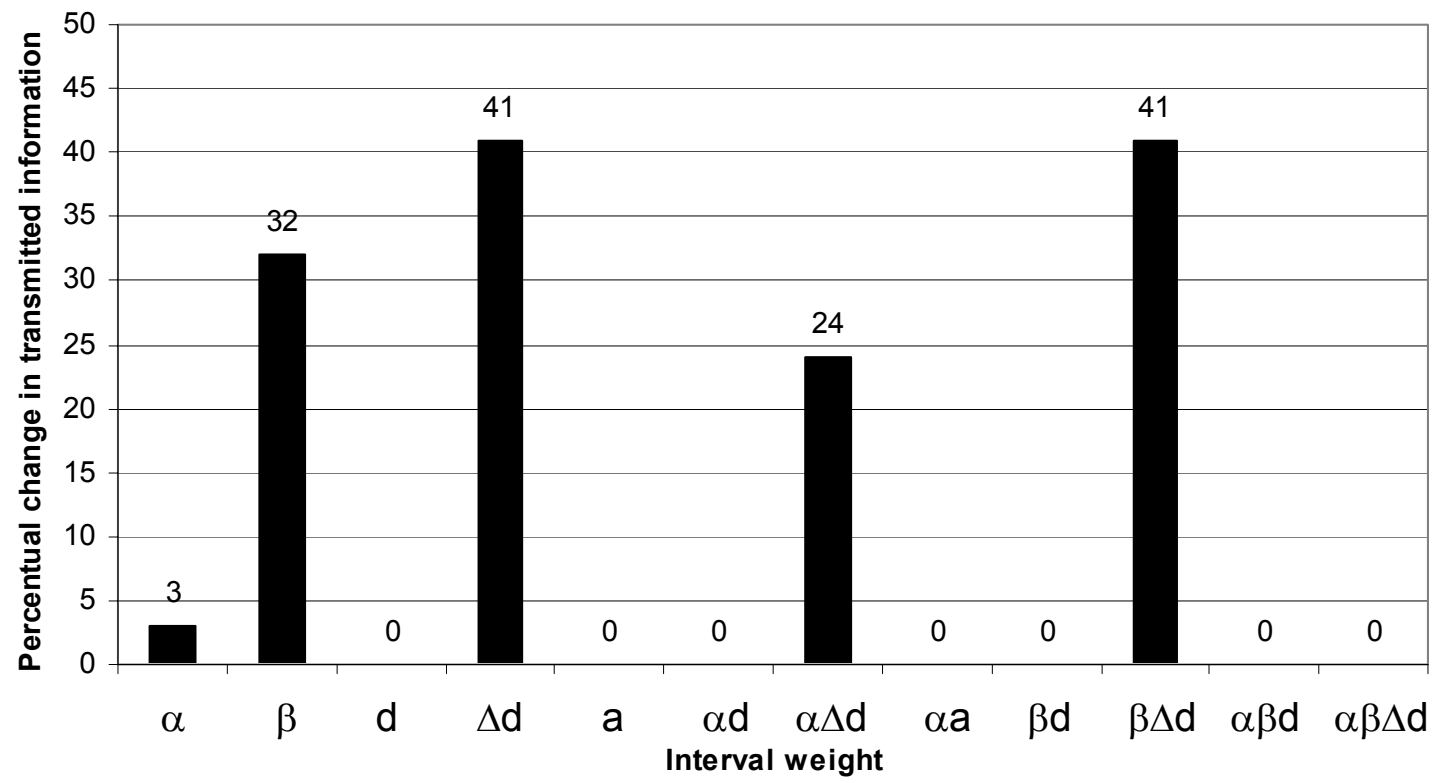

Fig. 15: Percentual change in the transmitted information obtained with each interval weight.

The weights associated with the terms $\Delta \mathrm{d}$ and $\beta \Delta \mathrm{d}$ affect the resulting signaling strategies most since the percentual change in the transmitted information compared to the reference strategy is $41 \%$. The weights of the terms $\beta$ and $\alpha \Delta d$ have also high contribution to the prioritization because $32 \%$ and $24 \%$ of the transmitted information differs from the reference strategy when varying these weights. It can be concluded that the weights related to the terms containing the approaching velocity and angle off attributes are the most important factors from among all the weights in the example air combat game. Thus, in the validation phase of the value function, one must concentrate on the assessment of these weights.

Although the presented sensitivity analysis gives significant validation information, similar analysis should be conducted in other air combat scenarios as well in order to be able to find out overall effects of the weights. On the other hand, the analysis is limited to what happens when only one weight varies at a time. The joint impact of two weights could be found out using a procedure in which two weights vary 
simultaneously and at the same time all other weights are kept at their point-estimates. For instance, the two most crucial weights, i.e, those related to the terms $\Delta \mathrm{d}$ and $\beta \Delta \mathrm{d}$, could be studied.

It should be noted that in addition to the weights, the shapes of the single attribute value functions affect signaling strategies. Naturally, the outcome of sensitivity analysis also depends on the single attribute value functions. The final assessment and validation of the value function is an iterative process that must be conducted in cooperation with pilots. In this process, different weights and shapes of the single attribute value functions must be tested by utilizing sensitivity analysis as well as by comparing signaling strategies obtained in various air combat games until satisfactory parameter values are identified.

\section{CONCLUSION}

We have presented a prioritization approach for selecting information that is transmitted within a team of pilots by the data link system during air-to-air combat. The preferences and goals of the pilots are modeled with a value function that is used for determining a team optimal signaling strategy with respect to the common goal of the team. Such a strategy gives the best sequence of the state information of the aircraft included in successive data link messages. The inherent uncertainty of the signaling problem is taken into account with interval analysis that allows the parameters of the value function as well as the state variables of the aircraft to vary within given ranges. The intervals can also be utilized in sensitivity analysis that gives important validation information especially about the weights of multiplicative value functions whose interpretation is less straightforward than in additive value functions.

We have carried out tests in which pilots have evaluated signaling strategies in different air combat scenarios with the IPAC software. In their opinion, the new approach gives better strategies than existing prioritization approaches implemented in the data link system. This conclusion is supported by the simulation examples of this paper. Hence, the presented prioritization approach seems to provide a possibility to improve the situation assessment capabilities of the pilots.

Although the preliminary validation results are promising, more analysis is needed before the implementation. One should study carefully how the shapes and the weights of the single attribute value functions affect the prioritization as well as how the transmitted information affects the decision making of the pilots. A possible approach is to include the prioritization approach into a dynamic multiplayer air combat game where the maneuvers of the players are selected optimally by using a decision making model. Analysis of this type could be carried out, e.g., with the help of existing batch air combat simulators (e.g., [16], [24]). By utilizing them, the impacts of signaling strategies on the outcome of the combat can be studied in a controlled and repeatable environment. On the other hand, the effect and value of extra information provided by data link messages could be analyzed with an influence diagram describing the decision making process of pilots [26], [27].

In practice, the new prioritization approach can be utilized in the implementation of the data link system as well as in a data link model included in decision making modules of air combat simulators. Although the approach is designed for the selection of the contents of data link messages, it can also be used, e.g., for prioritizing radio communications between computer guided aircraft in air combat simulators. Besides air combat, a similar approach could also be tailored to any problem where information is ranked in a dynamic, hostile, and uncertain environment and the most important information is being sent repeatedly via a network whose capacity is limited. 


\section{REFERENCES}

[1] A. Bagchi, and T. Basar, "Team Decision Theory for Linear Continuous-Time Systems," IEEE Transactions on Automatic Control, Vol. AC-25, No. 6, 1980, pp. 1154-1161.

[2] T. Basar, "An Equilibrium Theory for Multiperson Decision Making with Multiple Probabilistic Models," IEEE Transactions on Automatic Control, Vol. AC-30, No. 2, 1985, pp. 118-132.

[3] T. Basar, and G. Olsder, Dynamic Noncooperative Game Theory, 2nd ed., Academic Press, London, England, 1995, pp. 202-203.

[4] D. P. Bertsekas, Dynamic Programming and Optimal Control, Vol. 1, Athena Scientific, Belmont, MA, 1995, pp. 16-20.

[5] D. P. Bertsekas, Nonlinear Programming, Athena Scientific, Belmont, MA, 1995, pp. 361-382.

[6] K. C. Chu, "Team Decision Theory and Information Structures in Optimal Control Problems - Part II," IEEE Transactions on Automatic Control, Vol. AC-17, No. 1, 1972, pp. 22-28.

[7] W. Edwards, "How to Use Multiattribute Utility Measurement for Social Decisionmaking," IEEE Transactions on Systems, Man, and Cybernetics, SMC-7, 1976, pp. 326-340.

[8] W. Edwards, and F. H. Barron, "SMARTS and SMARTER: Improved Simple Methods for Multiattribute Utility Measurement," Organizational Behavior and Human Decision Processes, Vol. 60, 1994, pp. 306-325.

[9] A. Grace, Optimization Toolbox for Use with Matlab, User's Guide, The Math Works Inc., Natic, MA, 1995.

[10] Y. C. Ho, and K. C. Chu, "Team Decision Theory and Information Structures in Optimal Control Problems - Part I," IEEE Transactions on Automatic Control, Vol. AC-17, No. 1, 1972, pp. 15-22.

[11] M. Hura et al., "Interoperability: A Continuing Challenge in Coalition Air Operations," RAND, Santa Monica, Calif., RAND Documents, MR-1235-AF, 2000, pp. 107-121.

[12] R. P. Hämäläinen, and M. Pöyhönen, "On-line Group Decision Support by Preference Programming in Traffic Planning," Group Decision and Negotiation, Vol. 5, 1996, pp. 185-200.

[13] R. P. Hämäläinen, "Decisionarium - Aiding Decisions, Negotiating and Collecting Opinions on the Web," Journal of Multi-Criteria Decision Analysis, Vol. 12, Issue 2-3, 2004, pp. 101-110.

[14] R. L. Keeney, Value-Focused Thinking: A Path to Creative Decisionmaking, Harvard University Press, Cambridge, MA, 1992, ch. 5.

[15] R. L. Keeney, and H. Raiffa, Decision with Multiple Objectives: Preferences and Value Tradeoffs, Cambridge University Press, New York, 1993, ch. 3.

[16] E. Lazarus, "The Application of Value-Driven Decision-Making in Air Combat Simulation," Proceedings of the IEEE International Conference on Systems, Man, and Cybernetics, Orlando, FL, 1997, pp. 2302-2307.

[17] M. Lindstedt, R. P. Hämäläinen, and J. Mustajoki, "Using Intervals for Global Sensitivity Analyses in Multiattribute Value Trees," Multiple Criteria Decision Making in the New Millennium, Lecture Notes in Economics and Mathematical Systems, Vol. 507, M. Köksalan and S. Zionts, Eds., SpringerVerlag, Berlin, Germany, 2001, pp. 177-186.

[18] MATLAB User's Guide, The MathWorks Inc., Natick, MA, 1998.

[19] J. Mustajoki, and R. P. Hämäläinen, "Web-HIPRE: Global Decision Support by Value Tree and AHP Analysis," INFOR, Vol. 38, No. 3, 2000, pp. 208-220. Available: http://www.hipre.hut.fi

[20] J. Mustajoki, R.P. Hämäläinen, and A. Salo, "Decision Support by Interval SMART/SWING - A Method to Incorporate Uncertainty into Multiattribute Analysis," submitted for publication.

[21] A. Salo, and R. P. Hämäläinen, "Preference Assessment by Imprecise Ratio Statements," Operations Research, Vol. 82, No. 6, 1992, pp. 1053-1061.

[22] A. Salo, and R. P. Hämäläinen, "Preference Programming Through Approximate Ratio Comparisons," European Journal of Operational Research, Issue 3, Vol. 82, 1995, pp. 458-475. 
[23] A. Salo, and R. P. Hämäläinen, "Preference Ratios in Multiattribute Evaluation (PRIME) - Elicitation and Decision Procedures under Incomplete Information," IEEE Transactions on Systems, Man, and Cybernetics - Part A: Systems and Humans, Vol. 31, No. 6, 2001, pp. 533-545.

[24] P. Stehlin, I. Hallkvist, and H. Dahlstrand, "Models for Air Combat Simulation," Proceedings of the 19th ICAS Congress, ICAS-94-8.6.2, International Council of the Aeronautical Sciences, Les Mureaux, France, 1994, pp. 2190-2196.

[25] J. T. Sturdy, "Military Enhancements to Commercial Data Link Capabilities," Proceedings of the 19th IEEE Digital Avionics Systems Conference (DASC), Vol. 2, Philadelphia, PA, 2000, pp. 7E2/1 $7 \mathrm{E} 2 / 8$.

[26] K. Virtanen, T. Raivio, and R. P. Hämäläinen, "Decision Theoretical Approach to Pilot Simulation," Journal of Aircraft, Vol. 36, No. 4, 1999, pp. 632-641.

[27] K. Virtanen, T. Raivio, and R. P. Hämäläinen, "Modeling Pilot's Sequential Maneuvering Decisions by a Multistage Influence Diagram," Journal of Guidance, Control, and Dynamics, Vol. 27, No. 4, 2004, pp. 665-677.

[28] B. E. White, "Tactical Data Links, Air Traffic Management, and Software Programmable Radios," Proceedings of the 18th IEEE Digital Avionics Systems Conference (DASC), Vol. 1, St Louis, MO, 1999, pp. 5C5/1 - 5C5/8.

[29] H. Xu, and K. Mizukami, "Team-Optimal Closed-Loop Stackelberg Strategies for Discrete-Time Descriptor Systems," New Trends in Dynamic Games and Applications, Annals of the International Society of Dynamic Games, Vol. 3, G.J. Olsder, Ed., Birkhäuser, Boston, 1995, pp. 377-394. 